\title{
Article
}

\section{Investigating the properties of bio- chemical networks of artificial organisms with opposing behaviours}

\author{
Mokhtar, Maizura \\ Available at http://clok.uclan.ac.uk/7832/ \\ Mokhtar, Maizura ORCID: 0000-0003-0460-3696 (2013) Investigating the \\ properties of bio-chemical networks of artificial organisms with opposing \\ behaviours. Biosystems, - (-). ISSN 03032647
}

It is advisable to refer to the publisher's version if you intend to cite from the work. http://dx.doi.org/10.1016/j.biosystems.2013.03.011

For more information about UCLan's research in this area go to http://www.uclan.ac.uk/researchgroups/ and search for < name of research Group>.

For information about Research generally at UCLan please go to http://www.uclan.ac.uk/research/

All outputs in CLoK are protected by Intellectual Property Rights law, including Copyright law. Copyright, IPR and Moral Rights for the works on this site are retained by the individual authors and/or other copyright owners. Terms and conditions for use of this material are defined in the policies page.

\section{CLoK}

Central Lancashire online Knowledge www.clok.uclan.ac.uk

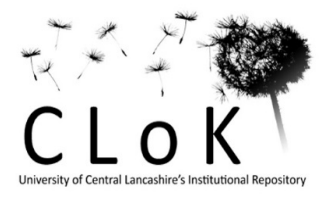




\title{
Investigating the Properties of Bio-Chemical Networks of Artificial Organisms with Opposing Behaviours
}

\author{
Maizura Mokhtar \\ School of Computing, Engineering and Physical Sciences, University of Central Lancashire, Preston, PR1 2HE, UK \\ (email: MMokhtar@uclan.ac.uk)
}

\begin{abstract}
Organisms, be it singled-celled organisms or multi-cellular organisms, are constantly faced with opposing objectives requiring different sets of behaviours. These behaviours can be classified into two, predatory behaviours or anti-prey behaviours, with one set of behaviours causing an opposite effect to the other. A healthy organism aims to achieve its equilibrium state or to be in homeostasis. Homeostasis is achieve when a balance between the two opposing behaviours is created and maintained. This raises some questions: is there an innate mechanism that encodes for these categories of behaviours? Is there also an innate mechanism(s) that resolves conflicts and allows switching between these two opposing behaviours? If we consider artificial organisms as single-celled organisms, how do the organisms' gene regulatory network, metabolic network and/or signalling network (their biochemical networks) maintain homeostasis of the organisms? This paper investigates the properties of the networks of best evolved artificial organisms, in order to help answer these questions, and guide the evolutionary development of controllers for artificial systems.
\end{abstract}

Keywords: artificial bio-chemical network, artificial genetic regulatory network, artificial organism, evolutionary algorithm

\section{Introduction}

An organism is constantly faced with the dilemma of balancing varying and opposing behaviours. This paper investigates the properties of bio-chemical networks of various artificial organisms, to investigate how their behaviours are encoded in the networks. Analysis of these properties may help us understand how a biological organism coordinates the switching of opposing behaviours. This understanding can also help us in the evolution of the bio-chemical networks for the artificial organisms.

The paper is divided into seven sections. Section 2 provides a brief understanding on how the opposing behaviours of the biological organisms are organised. In Sec. 3 , a number of questions that will be used to help guide the analysis to understand how the evolved bio-chemical networks of (artificial) organisms produced their behaviours are categorised. Section 4 describes what is the artificial bio-chemical network. Section 5 revisits the proposition that was made in the previous analysis of bio-chemical networks of artificial organisms (Mokhtar, 2012). Section 6 presents the results of analysis of other varying artificial organisms and network characteristics. Section 7 summarises and concludes this paper.

\section{Opposing predatory and anti-prey behaviours}

An organism's behaviours can be categorised into two distinct categories of behaviours, defined as either preda- tory or anti-prey behaviours.

\subsection{Multi-cellular organisms}

In multi-cellular organisms, predatory behaviours are to forage, fight and/or kill other animals in order to obtain food for the body. This category of behaviours typically requires high energy consumption and is considered high risk behaviours; but, to become a predator, one can lead to greater reward for the organism. For example, a carnivore (lion or tiger) would require high amount of energy to chase and capture a fast escaping deer (prey). This can cause exhaustion; but, the killed deer can provide greater reward in a form of extra food for the body. This will be different to when the carnivore choose to forage for carcases (dead animals) for food (this type of behaviour can be considered as anti-prey behaviour). This type of behaviour requires less energy (no chasing of prey is required) but only provides small reward to the animal.

A herbivore, for example, is performing predatory behaviour when it forages further from its nest in order to gather more food. The animal is considered as the predator and the environment is its prey. The animal may risk becoming prey to carnivores when foraging, but the reward is higher; in comparison to just staying to where the nest is at (this we considered as anti-prey behaviour ${ }^{1}$ ).

\footnotetext{
${ }^{1}$ We consider anti-predatory behaviour or behaviour in response
} 
A balance between the two opposing behaviours ensures that the organism is in homeostasis, a state important for the organism's survivability. If the animal is constantly favouring one behaviour in comparison to the other, this can significantly affect its survivability. If the carnivore favours (and unsuccessfully) capturing and killing its prey, the animal may exhaust its energy, leading to death ${ }^{2}$. At time of low energy, the organism may wish to forage for carcases in oder to preserve its energy. Similarly, if a herbivore choses to stay close to its nest, this can result in the significant drop in food availability near its nest. The animal may have insufficient food at times of high demand (for example, when there is significant change in environmental conditions that can reduce the animal's foraging capabilities).

Even in humans, these two categories of behaviours can be seen. Predatory behaviours are behaviours performed when we are at work and anti-prey behaviours are when we are at rest. We must perform both these categories of behaviour in moderation and in balance, because if we only concentrate on work, we will become exhausted and can damage our mental and physical health. But, if we only choose to rest, we will become obese and be affected by diseases caused by obesity.

We can further summarise that predatory behaviours are (pro-)active behaviours, with anti-prey behaviours as resistive, dormant and reactive behaviours.

\subsection{Single-celled organisms}

The categorisation of behaviours are not only limited to multi-cellular organisms. Single-celled organisms' behaviours can also be categorised into the two. We have indicated in (Mokhtar, 2012) that bacteriophage lambda ${ }^{3}$ can survive in two phages: either lysic, which we have considered as a predatory phage, or lysogenic, classified as the anti-prey phage. We have categorised the Bacteriophage lambda's behaviours as such because lysogenic is when the bacteriophage incorporates its DNA into the E. Coli's genome (its host) to ensure the survival of its species (anti-prey). Lysic is considered as predatory phage, because when the bacteriophage senses that its host $(E$. Coli) is weak, it will cause the destruction of its host in order for the bacteriophage to create new offsprings (Hasty et. al., 2001). If the bacteriophage remains lysogenic, the bacteriophage will die with its host. If the bacteriophage remains constantly lysic, this may reduce the availability of hosts, which in turn can affect the survival of its progeny.

to the presence of predation (Hegner, 1985), (Riechert and Hedrick, 1990), (Lima and Bednekoff, 1999) as predatory behaviour. This is because more energy is required when there is an increase in the awareness of the organism, since the organism must be well prepared to respond to an attack by the predator (Hegner, 1985), (Riechert and Hedrick, 1990).

${ }^{2}$ Chasing prey causes acid build up in the muscle (anaerobic). Excess acid build up can result in death for the animal.

${ }^{3}$ Bacteriophage lambda is not a single-celled organism but a virus; although its behaviours (be it simple behaviours) can be categorised into two: predatory or anti-prey.
Another example provided in (Mokhtar, 2012), is the flora bacteria residing in the gut. The flora bacteria survive in the hostile environment of the human gut by maintaining a symbiotic relationship with the gut. We have categorised the symbiotic relationship as an anti-prey behaviour and the high rate reproduction as the predatory behaviour. If the bacteria choses to predominantly perform one type of behaviours, the human immune system may produce a response that results in the destruction of its species (Rey et. al., 2006).

The two categories of behaviour can also be found in Dictyostelium discoideum, an example of an early multicellular organism ${ }^{4}$. As indicated in (Mokhtar, 2012), the collective (singular) behaviour of the Dictyostelium discoideum, for example, are to forage, fight and/or kill for food, in order to have sufficient food to form a collective morphology, move and reproduce in its environment. The former is considered as predatory behaviour and the later is anti-prey. If there are no balance between these two categories of behaviour, this may affect the survivability of the organism.

\section{Regulation of Behaviours}

The brain is responsible for the coordination of the two opposing categories of behaviour in multi-cellular organisms. In single-celled organisms, such coordination is provided directly by its gene regulatory network, or GRN, (de Jong, 2002), (Wolf and Eeckman, 1998). If we consider our artificial organism as a single-celled organism, we will be looking into how the GRN of the artificial organisms describe their behaviours. This will help us answer the following questions:

1. What encodes the organism's two different categories of behaviours? Can the behaviour be divided into predatory and anti-prey behaviours?

2. Is there an innate mechanism(s) that switches and resolves conflicts between the two opposing behaviours?

3. How do the organism's GRNs maintain homeostasis of the organism?

Lones et. al. (2011) have pointed out that in biological cells, the most significant interaction within the cells that underlies its behaviour is the interaction between its bio-chemical networks: "how the GRN indicates when and where proteins are expressed, which thereby determines which enzymes are present in the metabolic network and hence which reactions can take place within the cell". Essentially, the GRN is responsible in determining the cell's behaviour.

Therefore, we also ask:

\footnotetext{
${ }^{4}$ The Dictyostelium discoideum is considered as early multicellular organism because the Dictyostelium discoideum are a collection of individual single-celled organisms working collectively as one.
} 
1. How does an organism's GRN affect the metabolic network and/or signalling network of the organism in order to maintain homeostasis?

\section{Artificial Bio-chemical Network}

Lones et. al. (2011) proposed an artificial bio-chemical network architecture that couples an artificial genetic regulatory network to an artificial metabolic network.

\subsection{Artificial Genetic Regulatory Network}

The AGRN was formally defined by (Lones et. al., 2010) as $<G, L_{G}, I_{G}, O_{G}>$ and is presented in Algorithm 1 .

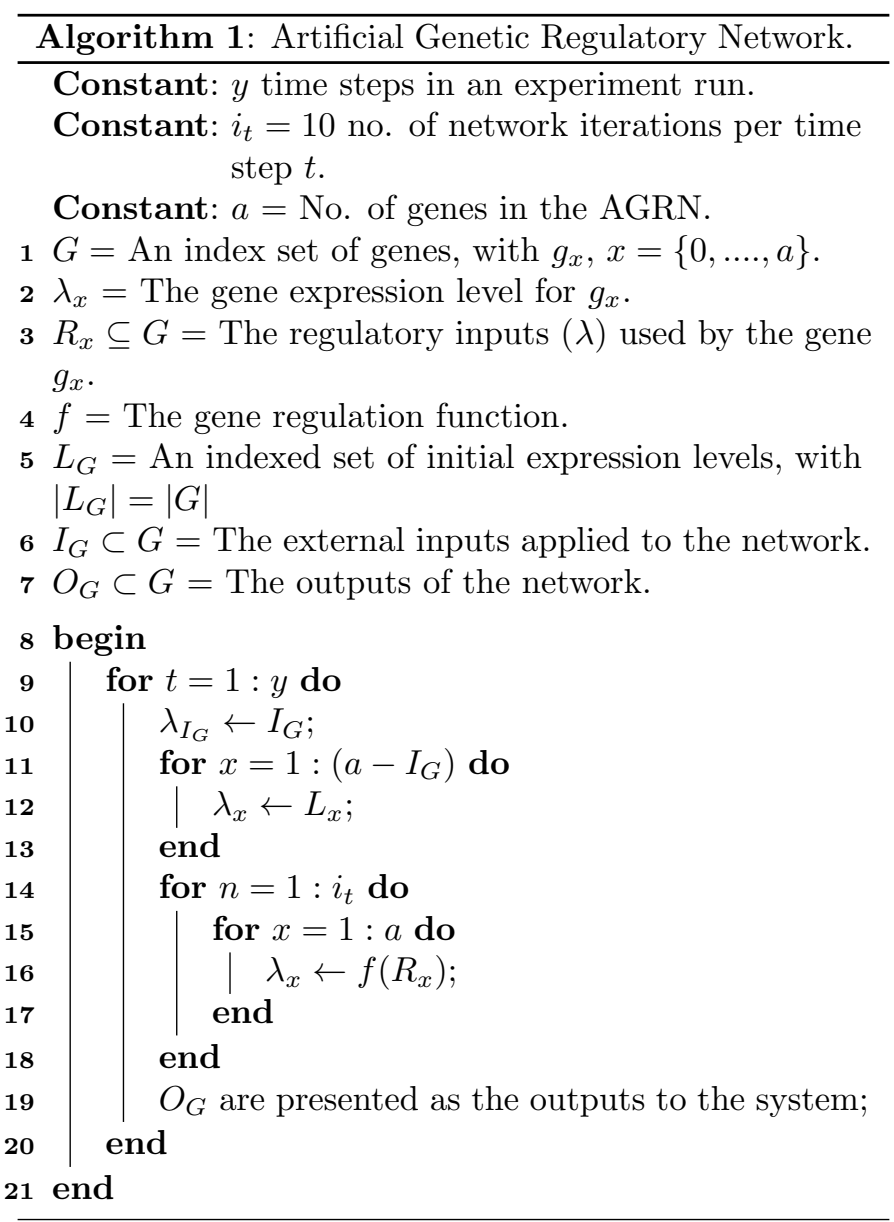

\subsection{Artificial Metabolic Network}

The AMN is formally described by (Lones et. al., 2011) as $A M N=<C, E, I_{E}, O_{E}>$ and is formulated using Algorithm 2.

\subsection{Coupled Artificial Bio-chemical Network or CABN}

The AGRN couples to the AMN using an injective coupling function $\chi: G_{C} \rightarrow E$. $G_{C} \subseteq G$ is the set of enzyme coding genes.

The AGRN is coupled to the AMN because in the biological coupled bio-chemical network, the GRN controls the

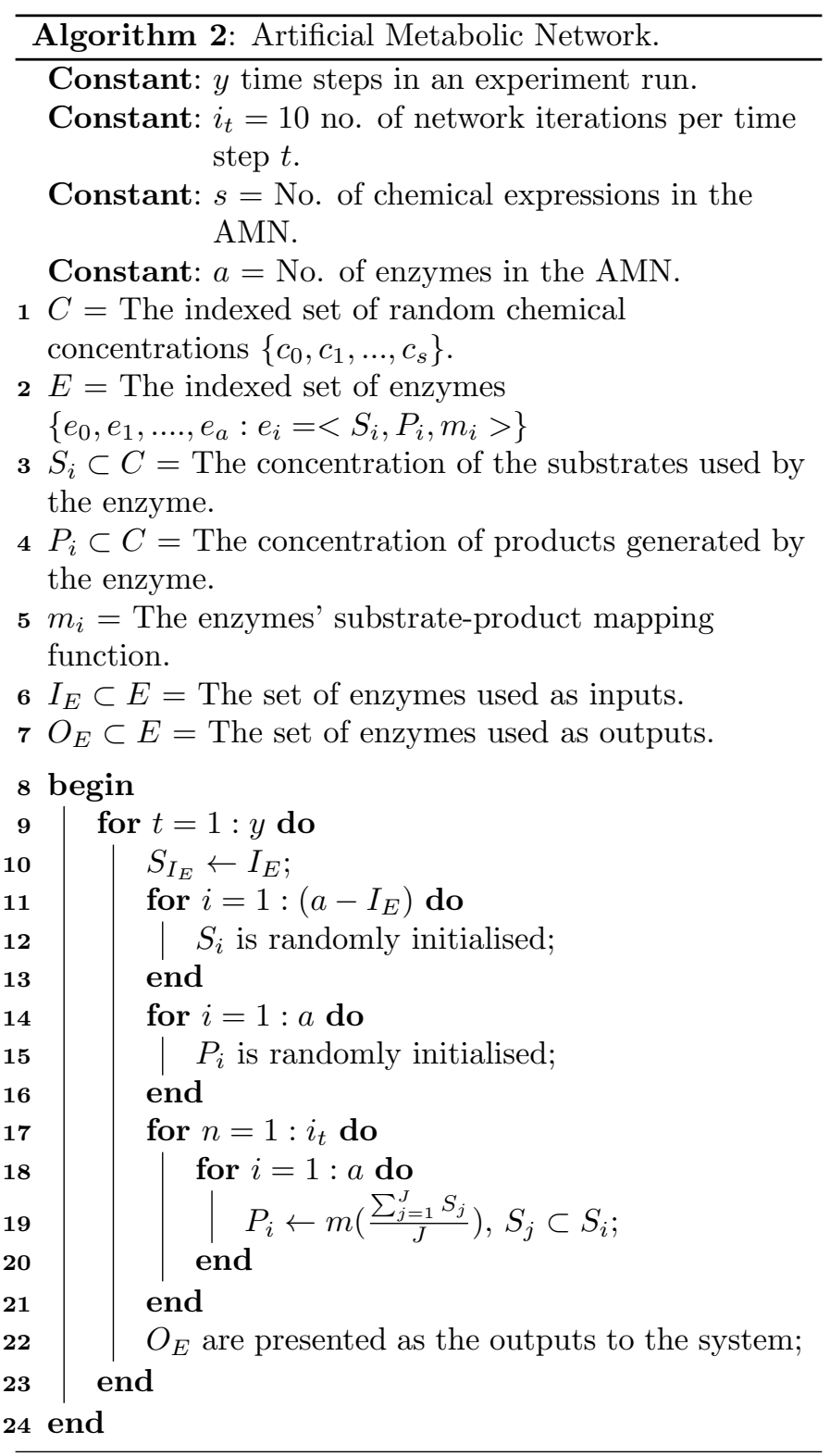

expression of the metabolic network (Lones et. al., 2011). The coupling of the AGN to the AMN is illustrated in Fig. 1 .

\section{Energy Management of an Artificial Organism using the ABN (Mokhtar, 2012)}

To help answer the questions posed in Sec. 3, a population of artificial bio-chemical networks $(\mathrm{ABN})$ were evolved, with the aim to manage the energy usage for an artificial organism. The objectives of the organism are:

1. to make use of the available energy resources $\left(E_{R}\right)$ to meet with their required energy demand $\left(E_{D}\right): U_{E}=$ $E_{R} \cdot C_{O 2}$

2. to save energy $\left(E_{S}\right)$ in storage $S_{E}$ at times when $E_{R}>$ $E_{D}: E_{S}=E_{R} \cdot\left(1-C_{O 2}\right)$ and $S_{E}=S_{E}+E_{S}$. 


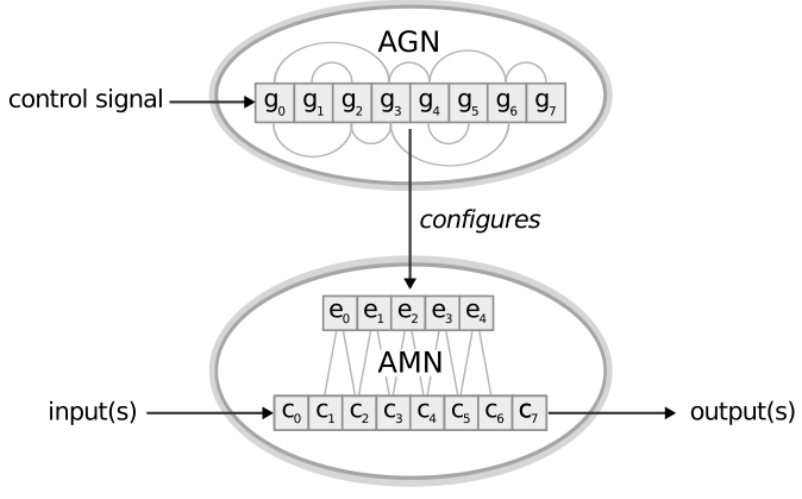

Figure 1: The coupled artificial bio-chemical network. Figure taken from (Lones et. al., 2011).

3. to use energy in $S_{E}$ to help meet with the demand $E_{D}$ when $E_{R}<E_{D}: U_{S}=S_{E} \cdot C_{O 1}$ and $S_{E}=S_{E}-U_{S}$.

The ABN consists of 6 genes in its AGRN, comprising of 54 weights $(w)$ and 6 slopes $(s)$. The 6 genes codes for 6 gene expression levels that lead to the production of 2 chemical outputs $C_{O 1}$ and $C_{O 2}$ ( $6 w$ and $2 s$ in its AMN). The chemical outputs govern the three listed behaviours: $U_{E}, E_{S}$ and $U_{S} . w \in[-1,1], s \in[-1,1]$ and $C_{O y} \in[0,1]$.

The values of $w$ and $s$ are evolved so that the best evolved ABNs achieved the fitness objectives of:

1. $E_{D} \approx\left(U_{E}+U_{S}\right)$

2. $S_{E}>0$

A population of 1000 ABN were evolved using SPEA2 (Strength Pareto Evolutionary Algorithm 2) developed by Popov (2005). The mutation rate $=0.0143$ and crossover rate $=0.5$. Network iteration $i_{t}=1$.

The evolved ABNs utilise the sigmoid function (1) for $f$ in the AGRN and $m$ in the AMN, respectively.

$$
f=\left(1+\exp ^{\left(-b-s \sum w z\right)}\right)^{-1}
$$

$z \subset \lambda$ for the AGRN and $z \subset S_{i}$ for the AMN.

The results of evolution were presented in (Mokhtar, 2012). Results indicated that in the evolved solutions, a balance or homeostasis between the two opposing behaviours can be achieved when the following characteristics within its $\mathrm{ABN}$ are observed (the output is $C_{O x} \in$ $[0,1])$ :

1. to make use of the available energy in $S_{E}$ to meet with the required $E_{D}\left(U_{S}\right)$ is considered as anti-prey behaviour. A behaviour is considered as anti-prey behaviour when its chemical output $C_{O x}$ is constantly $<0.5$.

2. to use $E_{R}$ to meet with the required $E_{D}\left(U_{E}\right)$ is considered as predatory behaviour. A behaviour is considered as predatory behaviour when its chemical output $C_{O x}$ is constantly $>0.5$.
3. $50 \%$ of the genes are predatory genes or are genes that are constantly producing $g_{x}>0.5$.

4. $25 \%$ of the genes are anti-prey genes or are genes that are constantly producing $g_{x}<0.5$.

5. $25 \%$ of the genes oscillate with $g_{x}$ mean of 0.5 .

Furthermore, Mokhtar (2012) indicated that:

1. The more positive weights $w$ within a gene/enzyme, the higher the likelihood for the gene/enzyme to be switched off $\left(C_{O x} / g_{x}=0\right)$.

2. The more negative weights $w$ within a gene/enzyme, the higher the likelihood for the gene/enzyme to be switched on $\left(C_{O x} / g_{x}=1\right)$.

3 . The more varied its weights $w$, the higher the likelihood that the gene/enzyme will become an oscillator.

These heuristics, as pointed out in (Mokhtar, 2012), indicate that the organism's behaviours can be described by the properties of its genes and chemicals produced by its ABN.

\section{Observations From ABNs Evolved to Solve Other Tasks}

To test if the heuristics presented in Sec. 5 and (Mokhtar, 2012) are also observed in other AGRNs and $\mathrm{ABNs}$, the properties of ABNs evolved to solve other tasks were analysed.

\subsection{Controlling Legged Robot Locomotion}

Lones et. al. (2011) presented the use of AGRNs (Sec. 6.1.1) and coupled ABNs (Sec. 6.1.2) for the purpose of controlling quadrupedal robot locomotion. The quadrupedal robot used by the authors is a simple robot design, comprising of a square top section with four legs connected by actuators at each corner. The actuators are limited to movement in the $\mathrm{x}$-axis plane, with a maximum elevation of $60^{\circ}$ from vertical.

\subsubsection{Artificial Genetic Regulatory Network}

The inputs to the AGRN are the four actuator angles of the robot, each presented to a gene $I_{G} \subset G$ at $n=0$. The outputs $\left(O_{G} \subset G\right)$ are used to set the torques of the actuators at every $n=i_{t}=10$ network iterations. The inputs are delivered via the initial gene expressions levels of $g_{0}, g_{1}, g_{2}$ and $g_{3}$, whilst the outputs are produced by $g_{6}, g_{7}, g_{8}$ and $g_{9}$.

The fitness value used in the evolution of the controller is the Euclidean distance of the robot from its start location (at $y=0$ ) to the location at $y=500$ time steps. Therefore, the higher the fitness value, the further the distance, the better the AGRN controller.

To help answer the questions presented in Sec. 3, the best populations evolved by (Lones et. al., 2011) were analysed $^{5}$. We analysed the populations by calculating

\footnotetext{
${ }^{5} 50$ populations, each evolved for 100 generations.
} 
the values of $\lambda$ for $G$ with $i_{t}=1$ and $L_{G}=1(\rho=\lambda$ when $f(1)) . \quad L_{G}=1$ was chosen because of the limited experiment data and information provided for the analysis, data and information which was used in the evolution of the controllers. $L_{G}=1$ was also chosen because this is the maximum input value that can be provided to the AGRN, therefore forcing the AGRN to operate at its most extreme conditions.

Because of this, a new definition of predatory and antiprey genes are also required. If $\rho_{x}=f_{g_{x}}(1)$ and $f_{g_{x}}(x)$ utilises the sigmoid function (1), predatory and anti-prey genes are defined as:

1. Predatory genes are genes with $\rho_{x} \geq 0.6$

2. Anti-prey genes are genes with $\rho_{x} \leq 0.4$

3. Neutral genes are genes with $0.4<\rho_{x}<0.6$

Figure 2 shows the attributes of the best evolved AGRN controllers. It shows that the best evolved controllers have equal numbers of predatory and anti-prey genes within the AGRN. Further investigation of the best evolved AGRN show similar symmetry in its AGRN. This is indicated in Fig. 3 to Fig. 5 .

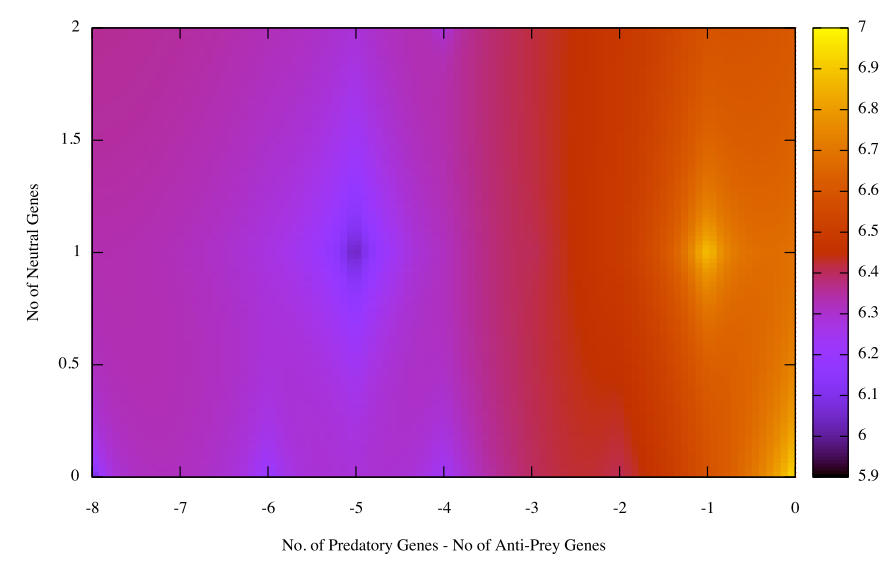

Figure 2: The attributes of the best evolved population of AGRN controllers. Symmetry is observed by the best evolved AGRNs, whereby the best evolved AGRNs have almost equal number of predatory and anti-prey genes. Predatory genes are genes with $\rho \geq 0.6$. Anti-prey genes are genes with $\rho \leq 0.4$. The colour values indicate the fitness value for a particular AGRN in the population (ignoring the 0.5 values in the $y$-axis). The higher the fitness value, the lighter the colour, the better the controller.

\subsubsection{Coupled Artificial Bio-Chemical Network or $C A B N$}

The objectives of the Coupled Artificial Bio-Chemical Network (CABN), different from the AGRN, is to walk as far as possible from the start location (similar to Sec. 6.1.1), and to return back to its start location when an external control signal is presented to the robot's CABN. The authors indicated that the best evolved controllers are coupled AGRNs with the sigmoid function (1) for $f$ and the Chirikov's Standard Map (2) for $m$ in the AMN.

$$
\begin{aligned}
& p_{n+1}=\left(p_{n}+K \sin \theta_{n}\right) \bmod 2 \pi \\
& \theta_{n+1}=\left(\theta_{n}+p_{n+1}\right) \bmod 2 \pi
\end{aligned}
$$

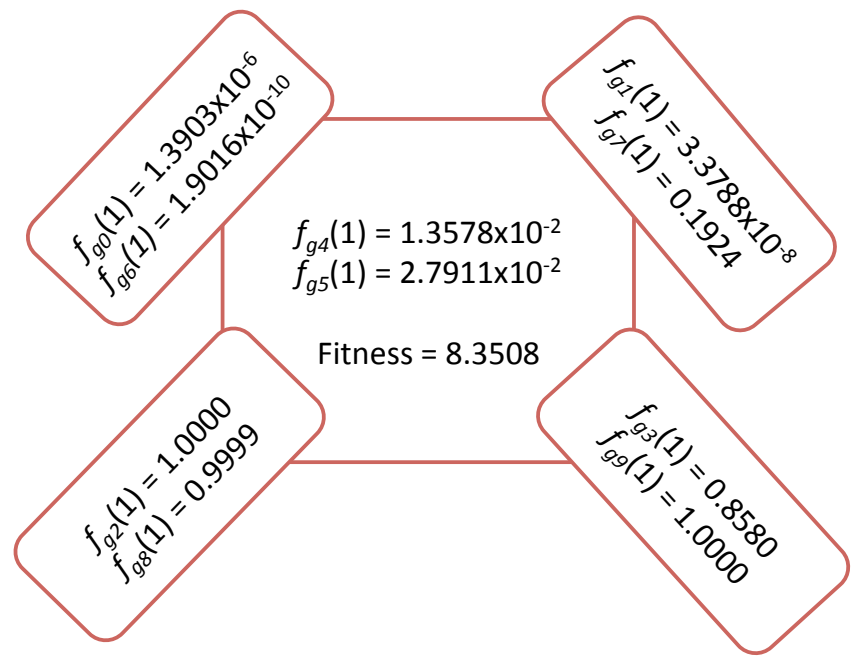

Figure 3: The values of $f_{g_{x}}(1)$ of the robot incorporated with the third best evolved solution. The values showed that symmetry is observed by the $O_{G}$ and $I_{G}$ in the (artificial) organism's AGRN.

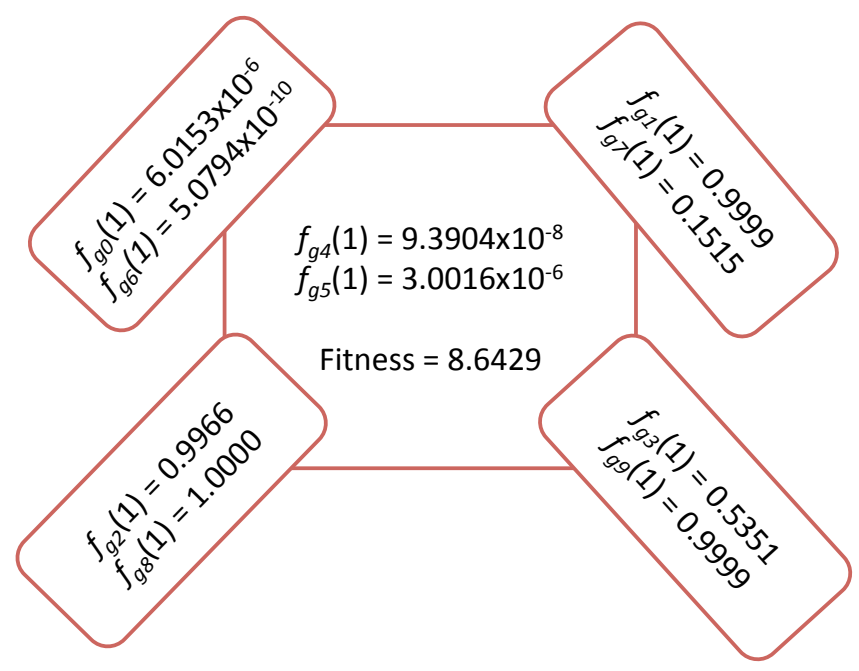

Figure 4: The values of $f_{g_{x}}(1)$ of the robot incorporated with the second best evolved solution. Symmetry is too observed by the $O_{G}$ and $I_{G}$ in the (artificial) organism's AGRN.

To investigate if the properties listed in Sec. 5 were observed in their best evolved controllers, their evolved CABNs' properties were analysed. We perform similar analysis techniques that were described in the previous section. We first observed the properties of the AGRN before looking at how the coupling between the two networks affects the robot's locomotion.

\subsubsection{CABN's Artificial Genetic Regulatory Network}

The Sigmoid AGRN properties are those described in Sec. 6.1.1. Section 5 described that in order for the artificial organism to provide for the required behaviours, its AGRN should consist of (i) $50 \%$ predatory genes, (ii) $25 \%$ anti-prey genes, and (iii) $25 \%$ neutral genes. There are 10 genes in each AGRN; therefore, the best evolved solutions should have 5 predatory genes, and 2 to 3 anti-prey and 


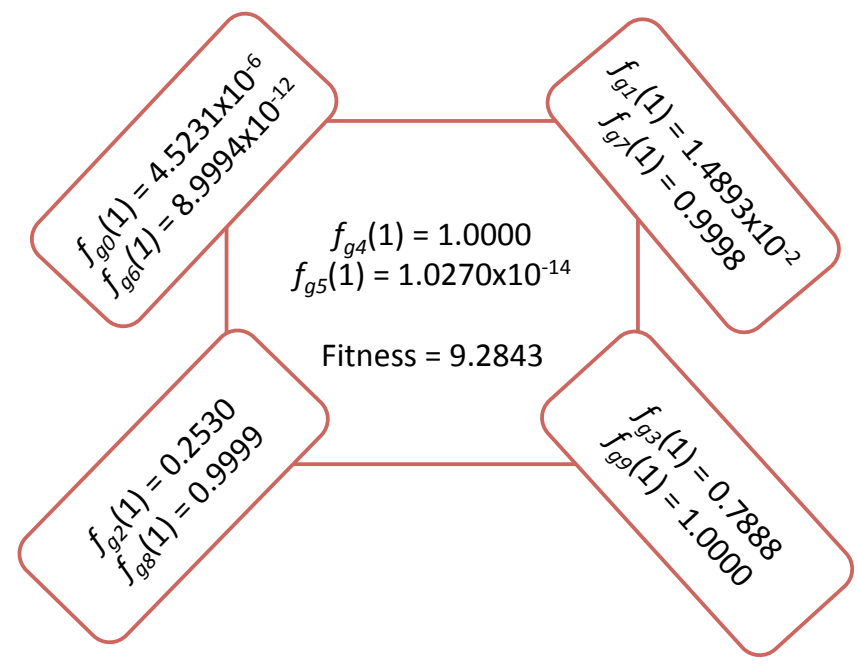

Figure 5: The values of $f_{g_{x}}(1)$ of the robot incorporated with the best evolved solution. Symmetry is too observed by the genes of the AGRN. This is because there is an almost equal number of predatory genes to anti-prey genes in the AGRN, with zero neutral gene in the AGRN.

neutral genes in their AGRN. There are also 10 chemical outputs produced by the AMN.

Figure 6 illustrates the properties of the best evolved population. This shows that best evolved CABNs have similar attributes to those listed in Sec. 5; with the best evolved controllers having $\sim 25 \%$ neutral genes (2-4 genes) and $\sim 50 \%$ to $70 \%$ predatory genes. We defined AGRNs with higher number of predatory genes as AGRNs that are prone towards predatory behaviours.

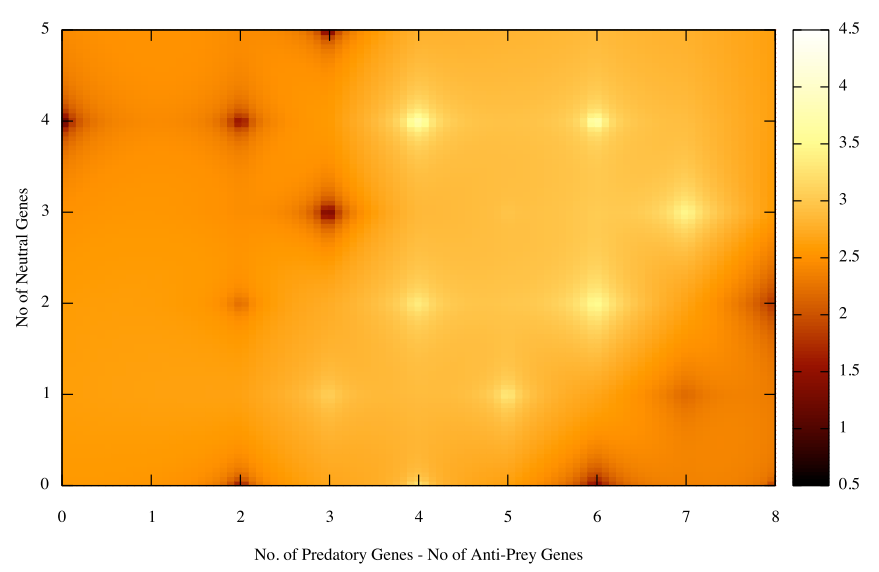

Figure 6: The properties of AGRNs from the best evolved populations. The higher the fitness value, the better the controller. There are 10 genes in the AGRN. The best evolved controllers have $\sim 25 \%$ neutral genes (2-4 genes) and $\sim 50 \%$ to $70 \%$ predatory genes. The colour values (see legend) indicate the fitness value attributed to a particular AGRN of the CABN.

\subsubsection{CABN's Artificial Metabolic Network}

The AGRN is coupled with a Chirikov's Standard Map AMN in order to provide for the required robot locomotion. To help us with our analysis, we will restrict our analysis to only the best evolved controllers. We will be analysing the chemical concentrations generated by the enzymes $\left(P_{i} \subset C\right)$ with:

1. The gene expression levels of the genes when $z=1$ (1)

2. The enzymes' initial conditions $S_{i}=1$

3. After network iteration $n=i_{t}=10\left(c_{i}(10)\right)$.

We define a predatory chemical as when $c_{i} \geq 0.6$, and an anti-prey chemical when $c_{i} \leq 0.4$. A neutral chemical has $0.4<c_{i}<0.6$.

Figures 7 - 10 illustrates the values produced by $g_{i}$ and $c_{i}$ after $n=i_{t}=10 . c_{0}-c_{3}$, each is presented with the actuator angle at its respective gait $\left(I_{E}\right)$ and $c_{6}-c_{7}$, each is its torque value $\left(O_{E}\right)$. Figures 7 - 10 indicated that, when $z=1$ (1) for each gene and the enzymes' initial conditions $S_{i}=1$, there are a total of $\approx 50 \%$ predatory genes + predatory chemicals in the CABN. The figures, therefore, show that the CABN has produced similar attributes to that of the previous system (Sec. 6.1.1 and in (Mokhtar, 2012)); whereby in the best evolved controllers, with $50 \%$ of the genes and chemicals produced by the CABN are categorised as predatory.

The predatory prone AGRN (due to the high number of predatory genes in the AGRN) is balanced by the AMN that produced $>50 \%$ anti-prey or neutral chemicals; hence symmetry within the network is observed.

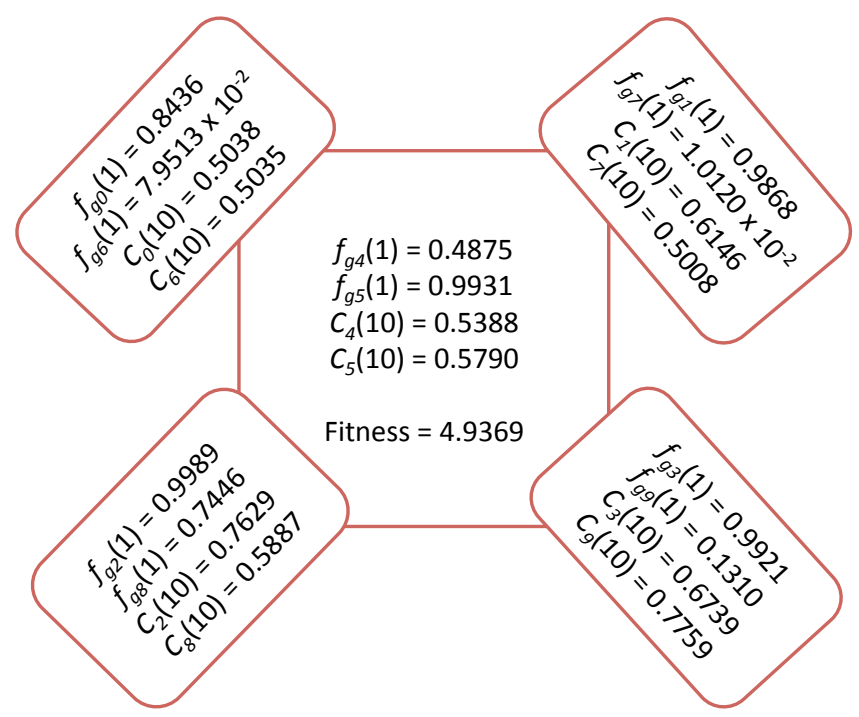

Figure 7: How symmetry is observed by the genes and enzymes in the (artificial) organism's CABN of the fourth best evolved solution. In its AGRN, there are 6 predatory genes, 3 anti-prey genes and 1 neutral gene. In its AMN, there are 4 predatory chemicals and 6 neutral chemicals produced. This suggests that the higher number of neutral chemicals in the AMN helps to balance the predatory bias found in the AGRN.

\subsubsection{Summary of Analysis}

Results of analysis on the AGRN and CABN evolved by (Lones et. al., 2011) indicated that the best AGRN and 


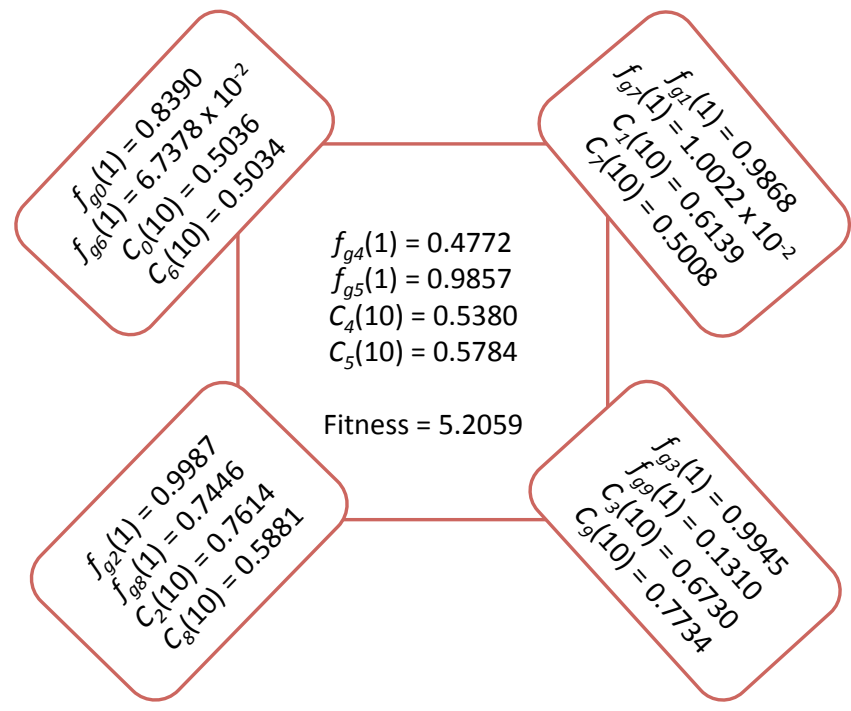

Figure 8: How the symmetry is observed by the genes and enzymes in the (artificial) organism's CABN of the third best evolved solution. There are 6 predatory genes, 3 anti-prey genes and 1 neutral gene, and there are 4 predatory chemicals and 6 neutral chemicals produced. The high number of neutral chemicals produced has help balanced the predatory tendencies provided by the predatory prone AGRN (that has higher number of predatory genes in comparison).

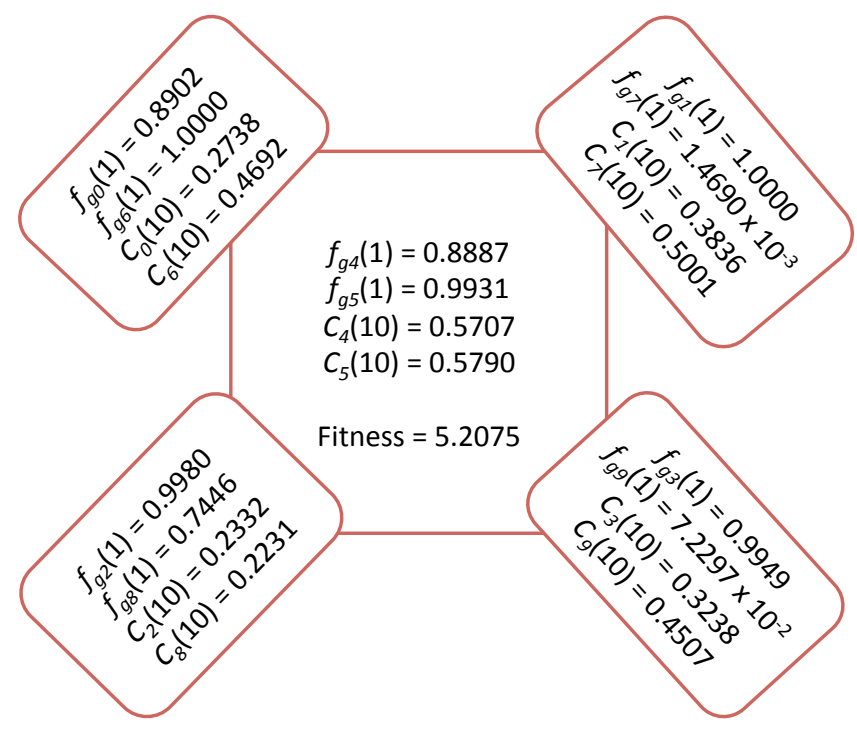

Figure 9: How the symmetry is observed by the genes and enzymes in the (artificial) organism's CABN of the second best evolved solution. There are 8 predatory genes and 2 anti-prey genes. There are 5 antiprey chemicals and 5 neutral chemicals produced, to help balance the predatory tendencies described by the predatory prone AGRN (higher number of predatory genes in comparison).

CABN controllers (controllers with the best fitness values) have:

1. Symmetry within its network; whereby the best evolved AGRN controllers have equal number of predatory and anti-prey genes in its AGRN and small number of neutral genes.

2. Similar to (Mokhtar, 2012), the best evolved CABN controllers' $\approx 50 \%$ predatory genes + chemicals are

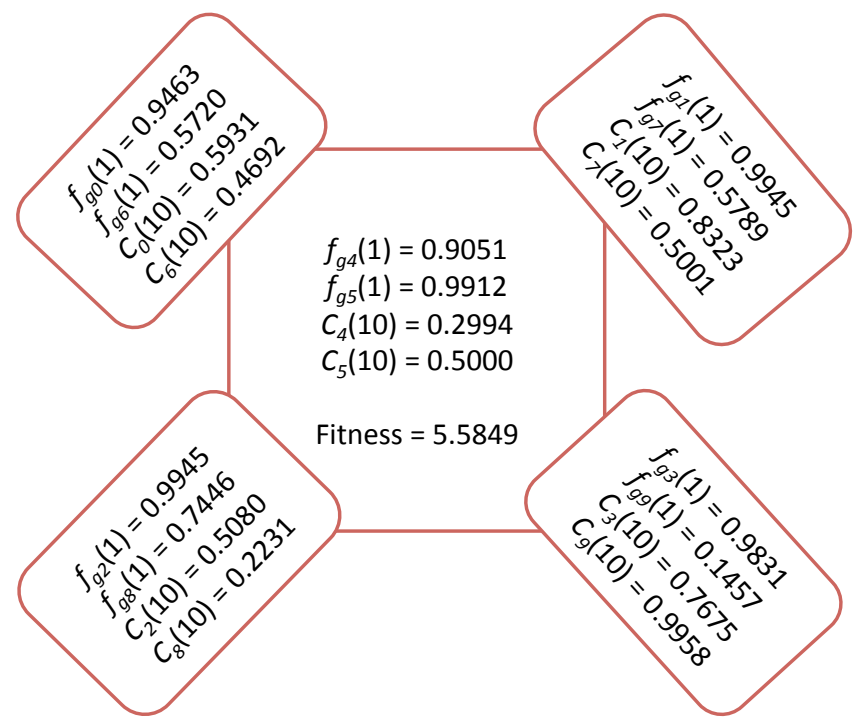

Figure 10: How the symmetry is observed by the genes and enzymes in the (artificial) organism's CABN of the best evolved solution. There are 7 predatory genes, 1 anti-prey genes and 2 neutral genes. There are 3 predatory chemicals, 2 anti-prey chemicals and 5 neutral chemicals produced. The predatory tendencies that is provided by the predatory prone AGRN is balanced by the high number of neutral chemical produced by the AMN.

balanced by the $\approx 50 \%$ anti-prey and neutral genes + chemicals.

\subsection{Similar Objectives to (Mokhtar, 2012)}

We revisited the system that was described in Sec. 5 and its objectives. We (re-)evolved the ABN and the AGRNonly controllers in order to investigate if the attributes listed are also observed in these systems, or whether they are similar to those described in Sec. 6.1. We will first discuss the attributes of the AGRN (Sec. 6.2.1) with $i_{t}=1$ and $i_{t}=10$, followed by the ABN (Sec. 6.2.2). The new evolved ABN has $i_{t}=10$.

\subsubsection{Artificial Genetic Regulatory Network}

We re-run the experiment described in (Mokhtar, 2012), but with only the AGRN (Sec. 4.1) providing the required controls. The population of 1000 AGRN was evolved using SPEA2 (Popov, 2005), with the mutation rate $=0.0143$ and crossover rate $=0.5$. The inputs $E_{D}, E_{R}$ and $S_{E}$ are presented to all genes, and $g_{x}$ to $g_{x-(d-1)}$ are the output genes with $d$ is the number of output and $x$ is the number of genes in the AGRN.

For $n=1$, we first evolved using the same fitness objectives listed in (Mokhtar, 2012). The AGRNs are first evolved so that the best evolved AGRNs achieved the fitness objectives of (Algorithm 3):

1. $E_{D} \approx\left(U_{E}+U_{S}\right)\left(F_{1}\right)$

2. $S_{E}>0\left(F_{2}\right)$

The population is evolved for 1000 generations. The AGRN differs from the previous, whereby each AGRN has 


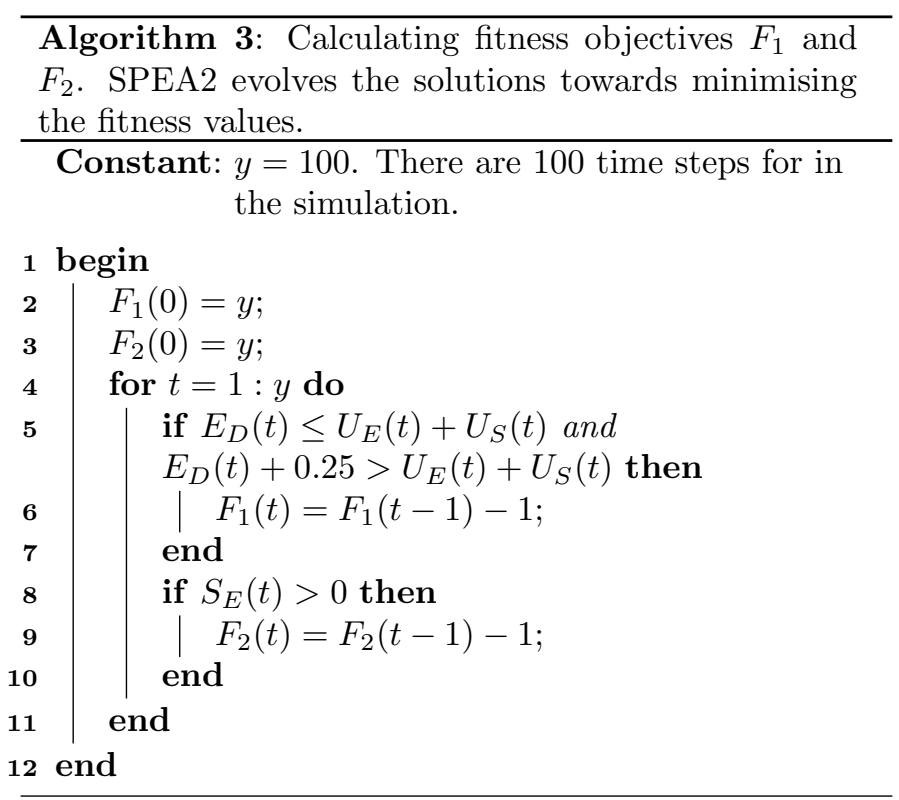

$d=3$ output genes instead of 2 . Each of the output genes is responsible for:

1. to make use of the available $E_{R}$ to meet with the required $E_{D}: U_{E}=E_{R} \cdot g_{x} \cdot x$ is the number of genes in the AGRN.

2. to save energy $\left(E_{S}\right)$ in $S_{E}: E_{S}=E_{R} \cdot g_{x-1}$ (instead of $E_{S}=E_{R} \cdot\left(1-g_{x}\right)$, if $\left.d=2\right)$.

3. to use energy in $S_{E}$ to help meet with $E_{D}: U_{S}=$ $S_{E} \cdot g_{x-2}\left(\right.$ instead of $U_{S}=S_{E} \cdot g_{x-1}$, if $\left.d=2\right)$.

Mokhtar (2012) indicated that:

1. $U_{E}$ provided by $g_{x}$ is considered as predatory behaviour.

2. $U_{S}$ provided by $g_{x-2}$ if $d=3$ or $g_{x-1}$ if $d=2$ is considered as anti-prey behaviour.

Because of the third output gene, another fitness objective is added, which states the population is to evolve so that:

1. $g_{x-1}+g_{x} \leq E_{R}\left(F_{0}\right)$.

Two definitions of predatory and anti-prey genes are presented:

1. Mokhtar (2012) states that:

(a) Predatory genes are genes that are constantly producing the output $\approx 1$

(b) Anti-prey genes are genes that are constantly producing the output $\approx 0$

(lines 3 - 21 in Algorithm 4)

2. In Sec. 6.1, we stated that if $\rho_{x}=f(1)$ and $f_{x}(1)$ utilises the sigmoid function (1):

(a) Predatory genes are genes with $\rho_{x} \geq 0.6$

(b) Anti-prey genes are genes with $\rho_{x} \leq 0.4$

(lines 3 - 11 in Algorithm 5)
Algorithm 4: Calculating fitness objectives $F_{3}$ to $f_{6}$. SPEA2 evolves the solutions towards the minimised fitness values.

Constant: $y=100$. There are 100 time steps for in the simulation.

Constant: $x=$ Number of genes $(g)$ in the AGRN.

Input $: \lambda_{i}=$ The gene expression level for $g_{i}$; $i \in x$.

Output : $g_{x} \subset G=$ Output gene responsible for $U_{E}$.

Output $: g_{(x-1)} \subset G=$ Output gene responsible for $U_{S}$.

1 begin

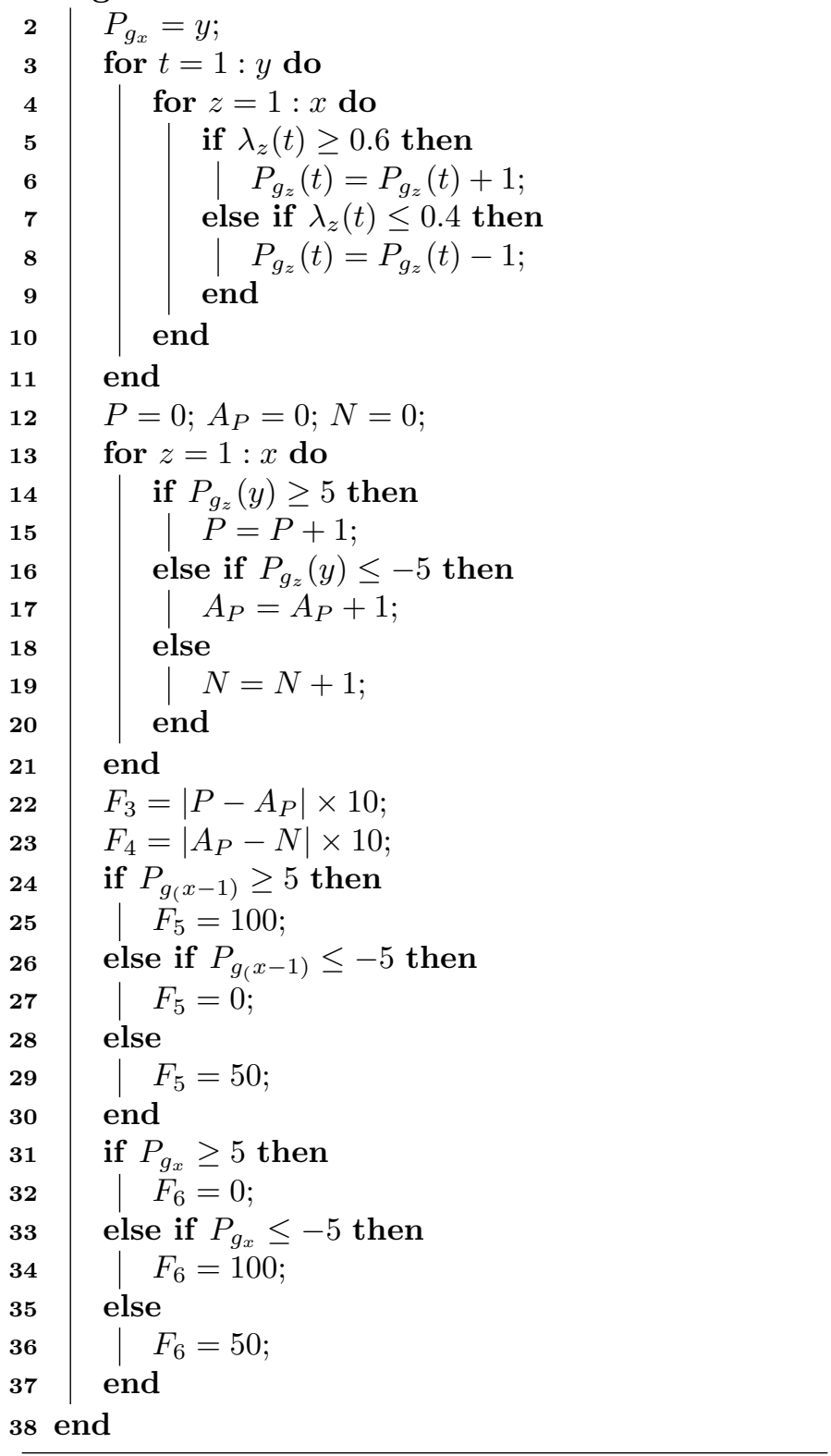

The best evolved solution have the fitness values of $F_{1}=$ $30, F_{2}=0$ and $F_{0}=57$.

1. If based on the definition of predatory and anti-prey genes described in (Mokhtar, 2012), the best evolved 


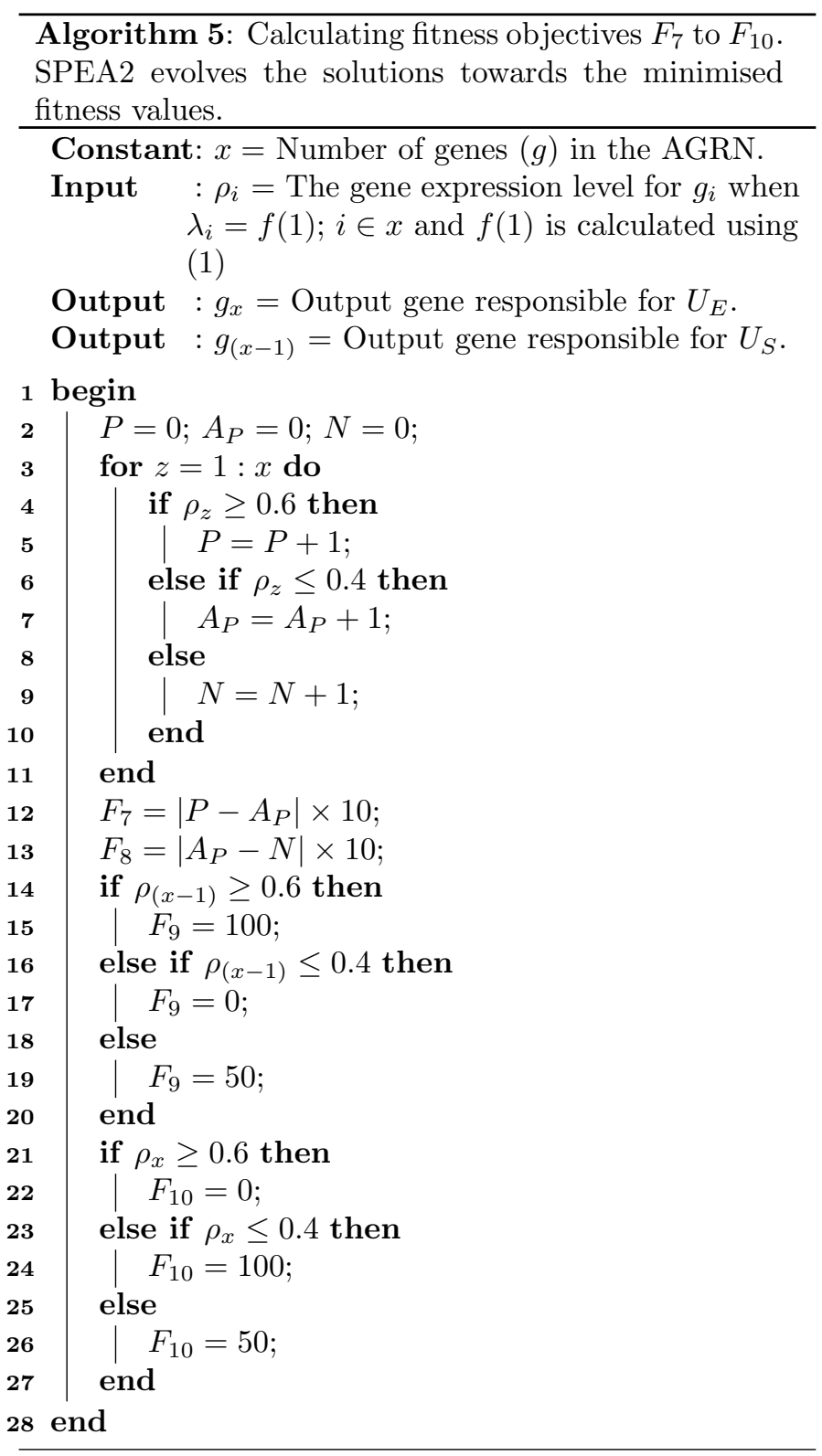

AGRN has 6 predatory genes, 5 anti-prey genes and 1 neutral genes ${ }^{6}$. Results also show that in the best evolved AGRN:

(a) $g_{x}$ is categorised as a predatory gene,

(b) $g_{x-1}$ is categorised as a neutral gene, and

(c) $g_{x-2}$ is categorised as an anti-prey gene.

These categorisation of behaviours are similar to the observation made by (Mokhtar, 2012).

2. If based on the definition of predatory and anti-prey genes described in Sec. 6.1, this best evolved AGRN instead has 1 predatory genes, 4 anti-prey genes and 8 neutral genes. Results also show that in the best evolved AGRN:

${ }^{6}$ These characteristics are similar to the systems discussed in Sec. 6.1 (the characteristics of the best evolved AGRNs and CABNs used to control the legged robot).
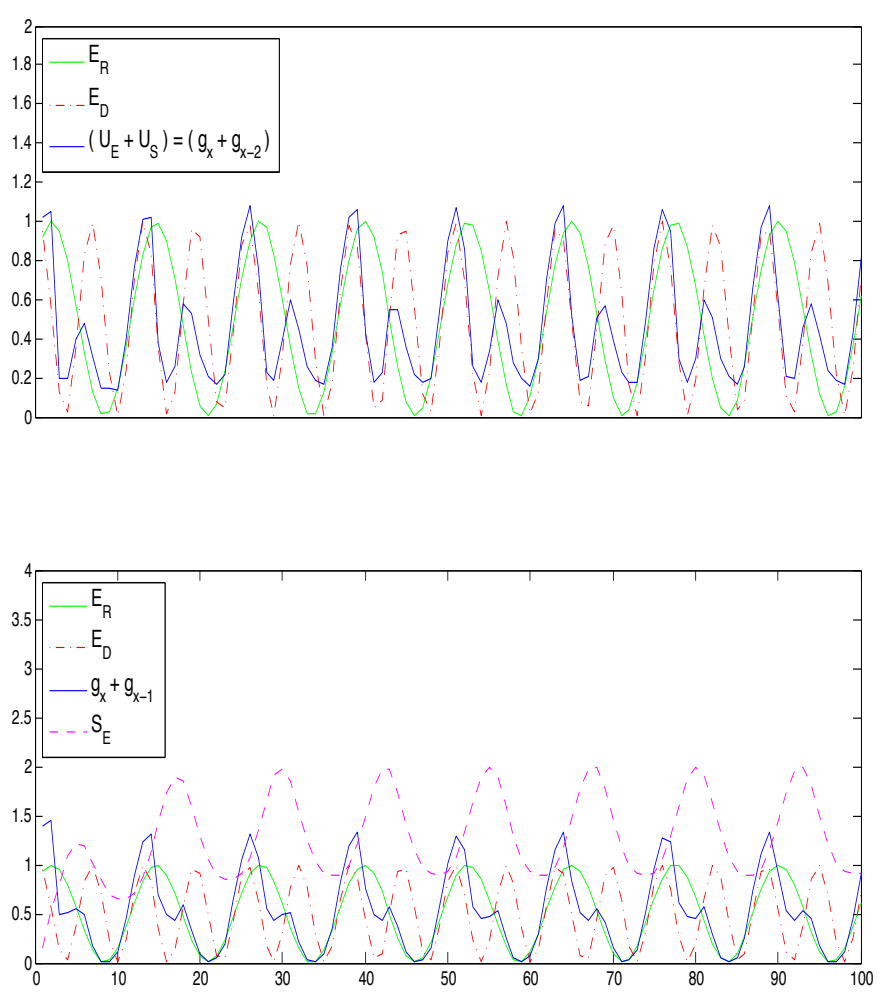

Figure 11: The $U_{E}, U_{S}, E_{S}, g_{x}, g_{x-1}$ and $g_{x-2}$ for the best evolved AGRN from Set 1. This best evolved AGRN has the fitness values of $F_{1}=30, F_{2}=0$ and $F_{0}=57$. The top figure shows the outputs of the AGRN aiming to meet $E_{D}$. The bottom figure, however, shows the AGRN violates the rule $g_{x-1}+g_{x} \leq E_{R}$, whilst doing so.

(a) $g_{x}$ is categorised as a predatory gene,

(b) $g_{x-1}$ is categorised as a neutral gene, and

(c) $g_{x-2}$ is categorised as an anti-prey gene;

Similar observations are also described in (Mokhtar, 2012).

The best evolved AGRN, however, violates the rule $g_{x}+$ $g_{x-1} \leq E_{R}$. This is as shown in Fig. 11 .

To help improve the outcome of the evolution, a second population of AGRNs are evolved using the fitness objectives $F_{1}$ and $F_{2}$, as well as new fitness objectives derived based on the analysis presented in (Mokhtar, 2012) $\left(F_{3}\right.$ and $\left.F_{4}\right)$. The new fitness objectives state that there should be almost equal number of neutral and anti-prey genes in the AGRN (lines $22-23$ in Algorithm 4) ${ }^{7}$. Similar to (Mokhtar, 2012), the AGRN has $d=2$ output genes.

The population was also evolved for 1000 generations. The best fitness values produced after the 1000th generation are $F_{1}=37$ and $F_{2}=1$.

1. If based on (Mokhtar, 2012), the best evolved AGRN has 4 predatory genes, 5 anti-prey genes and 3 neutral genes. $g_{x}$ and $g_{x-1}$ are both categorised as predatory genes.

\footnotetext{
${ }^{7}$ The population was evolved with $F_{3}$ because of the contradicting observation presented in Sec. 6.1.1
} 


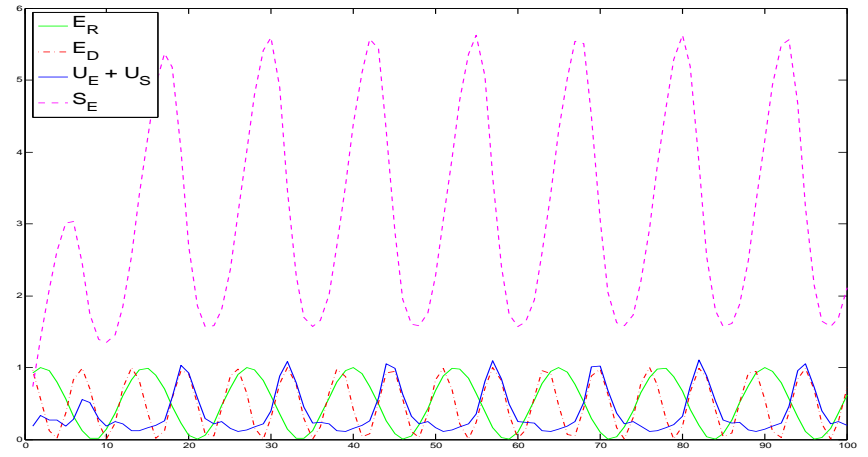

Figure 12: The $U_{E}$ and $U_{S}\left(g_{x}\right.$ and $\left.g_{x-1}\right)$ for the best evolved AGRN from Set 2. This best evolved AGRN has the fitness values of $F_{1}=37$ and $F_{2}=1$.

2. If based on Sec. 6.1, this best evolved AGRN has 3 predatory genes, 5 anti-prey genes and 4 neutral genes. $g_{x}$ is categorised as a predatory gene and $g_{x-1}$ is categorised as an anti-prey gene.

The best evolved AGRN has more anti-prey genes than predatory genes. This causes the organism to save as much energy as possible $\left(U_{S} \rightarrow S_{E}\right)$, to use at time when the energy resources $\left(E_{R}\right)$ is low. This is as shown in Fig. 12. These characteristics were too observed by Mokhtar (2012).

A third population was evolved using the fitness objectives $F_{1}$ to $F_{4}$, as well as a new set of fitness objectives derived based on the analysis of the best evolved AGRNs and ABNs described in Sec. $6.1\left(F_{5}\right.$ to $\left.F_{10}\right)$. The fitness objectives state that:

1. $g_{x-1}$ is considered as anti-prey $\left(F_{5}\right)$, lines $24-30$ in Algorithm 4.

2. $g_{x}$ is considered as predatory genes $\left(F_{6}\right)$, lines $31-37$ in Algorithm 4.

3. Almost equal number of predatory and anti-prey genes in the AGRN given $\rho=f(1)\left(F_{7}\right.$ and $\left.F_{8}\right)$, lines $3-13$ in Algorithm 5.

4. $\rho_{x-1}$ is considered as anti-prey genes $\left(F_{9}\right)$, lines 14 20 in Algorithm 5.

5. $\rho_{x}$ is considered as predatory genes $\left(F_{10}\right)$, lines $21-27$ in Algorithm 5.

The population is evolved for 1000 generations $^{8}$, and there are two output genes in the AGRN similar to (Mokhtar, 2012).

The best evolved solution has the fitness values $F_{1}=23$ and $F_{2}=0$. The AGRN:

1. if based on (Mokhtar, 2012), the best evolved AGRN has 6 predatory genes, 6 anti-prey genes and 0 neutral genes. $g_{x}$ is categorised as a predatory gene and $g_{x-1}$ as an anti-prey gene.

\footnotetext{
${ }^{8}$ The last population of the previous evolution was used as the initial population.
}

2. if based on Sec. 6.1, this best evolved AGRN has 6 predatory genes, 6 anti-prey genes and 0 neutral genes. $g_{x}$ is categorised as a predatory gene and $g_{x-1}$ is categorised as an anti-prey gene.

The symmetry in this best evolved AGRN is similar to that of the best evolved AGRNs and CABNs used to control the legged robots (Sec. 6.1). The characteristics of the last evolved population for the third set, illustrated in Fig. 14, indicate the similarity and symmetry. This best evolved AGRN is also best among the three sets because its $U_{E}+$ $U_{S}$ values match closer to its $E_{D}$ (Fig. 11 to Fig. 13)

The results also show that, with the introduction of the proposed fitness objectives $F_{3}-F_{10}$, better evolved solutions can be produced. This combination of fitness objectives are used from this point forth.

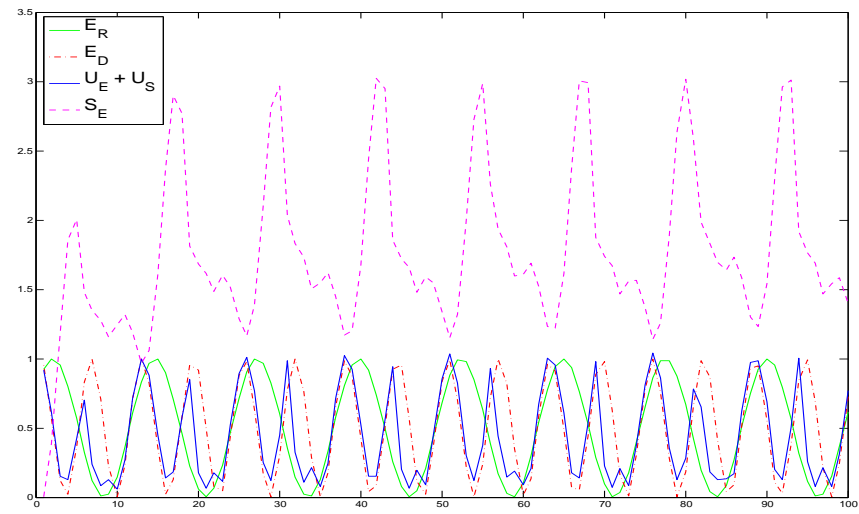

Figure 13: The $U_{E}$ and $U_{S}\left(g_{x}\right.$ and $\left.g_{x-1}\right)$ for the best evolved AGRN from Set 3. This best evolved AGRN has the fitness values of $F_{1}=23$ and $F_{2}=0$.

For the AGRN with $i_{t}=10$, the population were evolved using the fitness objectives presented in Algorithms 4 and 5 $\left(F_{3}-F_{10}\right)$. After 1000 generation, the best evolved AGRN has the fitness value of $F_{1}=41$ and $F_{2}=0$.

1. If based on (Mokhtar, 2012), the best evolved AGRN has 4 predatory genes, 4 anti-prey genes and 4 neutral genes. $g_{x}$ is categorised as a predatory gene and $g_{x-1}$ as an anti-prey gene.

2. If based on Sec. 6.1, this best evolved AGRN has 4 predatory genes, 6 anti-prey genes and 2 neutral genes. $g_{x}$ is categorised as a predatory gene and $g_{x-1}$ is categorised as an anti-prey gene.

Figure 16 illustrates how well this best evolved AGRN's $U_{E}+U_{S}$ aims to meet its $E_{D}$ given the $E_{R}$. The figure shows that the best evolved organism is unable to meet $E_{D}$ most effectively. This is because it refuses to use its energy in $S_{E}$ when $E_{D}>E_{R}$ most appropriately. If based on the observation made by (Mokhtar, 2012), these behavioural tendencies are the result of the inequality in the AGRN due to the higher number of anti-prey + neutral genes.

The characteristics of the last evolved population is shown in Fig. 15. The figure does however show similar 


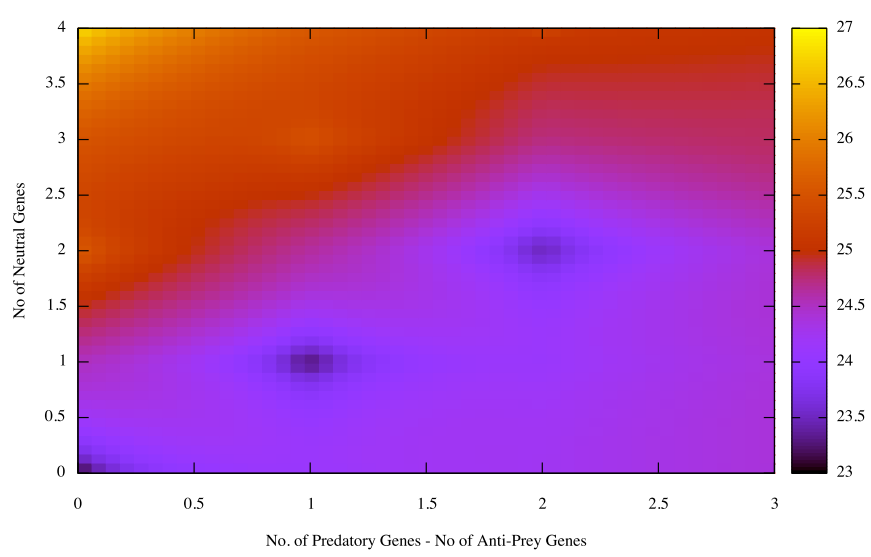

(a) Derived based on the method of analysis described in Sec. 6.1 (Algorithm 5)

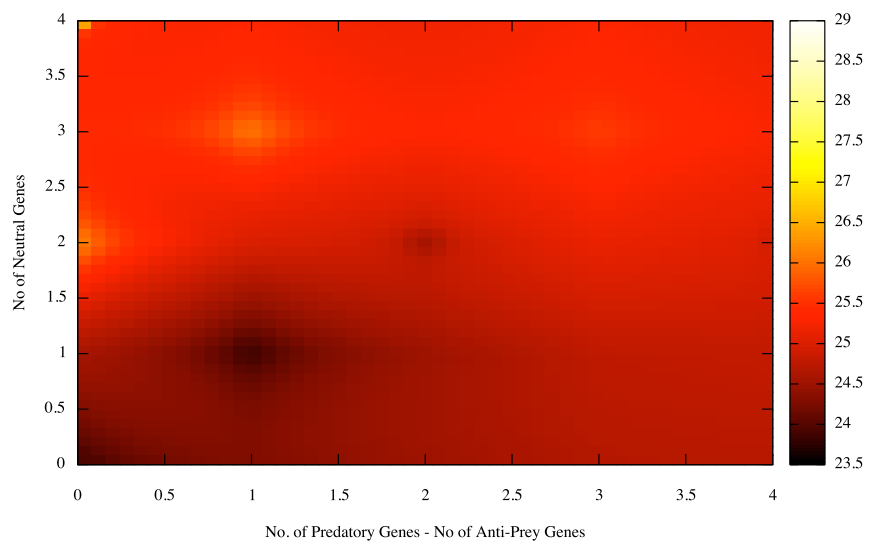

(b) Derived based on the method of analysis described in (Mokhtar, 2012)

Figure 14: The properties of AGRNs from the best (and last) evolved population of the third set. The colour values (legend) indicate the fitness value $F_{1}$ for a particular AGRN $\left(F_{2}<5\right)$. The lower the fitness value, the better the (artificial) organism. Figures show that the best evolved organism has symmetrical property in its AGRN, with an almost equal number of predatory and anti-prey genes, and low number of neutral genes (ignoring the 0.5 values in the y-axis). This observation is similar to the results of analysis described in Sec. 6.1 .

characteristics to that described in Sec. 6.1, which shows that good solutions (solutions with low fitness values) have an almost equal number of predatory and anti-prey genes and low number of neutral genes in its AGRN (Fig. 17).

\subsubsection{Artificial Bio-chemical Network}

We re-run the experiment described in (Mokhtar, 2012), but with $i_{t}=10$ and the fitness objectives $F_{1}$ to $F_{10}$. A population of $750 \mathrm{ABN}$ was evolved using SPEA2 (Popov, $2005)$, with the mutation rate $=0.0143$ and crossover rate $=0.5$, for 1000 generations.

The evolutionary process has produced the best evolved ABN with similar characteristics to that of the previous systems. The best evolved solution with fitness values of $F_{1}=32$ and $F_{2}=0$ has:

1. If based on the categorisation of behaviours described

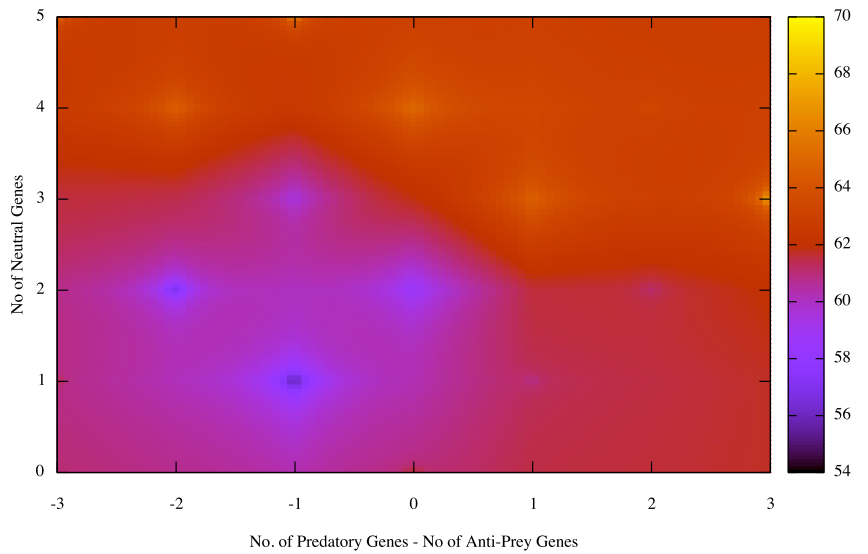

(a) Derived based on the method of analysis described in Sec. 6.1 (Algorithm 5)

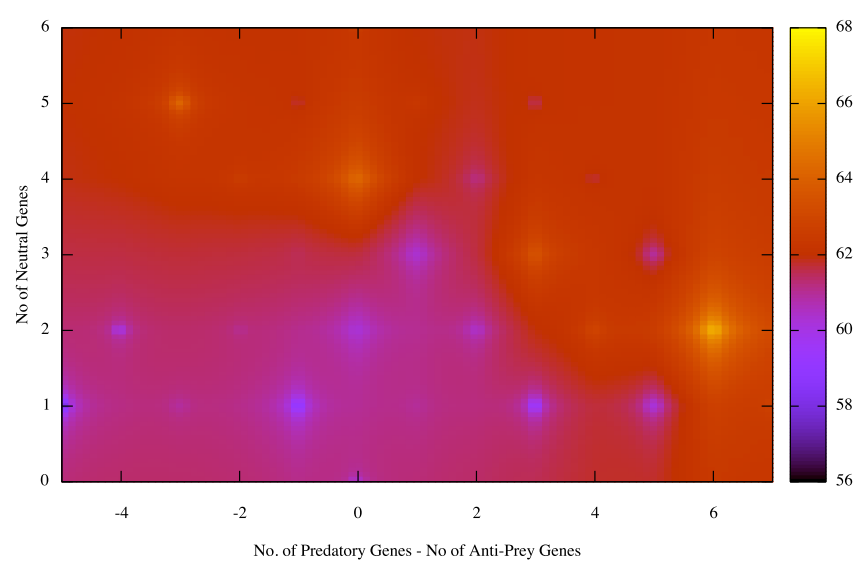

(b) Derived based on the method of analysis described in (Mokhtar, 2012)

Figure 15: The properties the best evolved population of the AGRNs with $i_{t}=10$. The colour values (legend) indicate the fitness value $F_{1}$ for a particular AGRN $\left(F_{2}<5\right)$. The lower the fitness value, the better the (artificial) organism. Figures show that the best evolved organism has more anti-prey genes in comparison to the predatory. genes. This has resulted in the best evolved solution having greater tendencies to store energy at time when $E_{D}<E_{R}$, and not to use the energy when $E_{D}>E_{R}$ (Fig. 16). Figures also show that when there is an almost equal number of predatory and anti-prey genes and low number of neutral genes, the fitness value is low, which indicates for a good solution (Fig. 17).

by (Mokhtar, 2012), there are 3 predatory genes, 3 anti-prey genes and 0 neutral genes, with

(a) $C_{O 1}$ used to decide on the $U_{S}$ (which indicates how to utilise the available energy in $S_{E}$ to meet with the required $E_{D}$ ) is categorised as neutral.

(b) $C_{O 2}$ that decides on the $U_{E}$ (how to use $E_{R}$ to meet with the required $\left.E_{D}\right)$ is considered as neutral

2. If is based on Sec. 6.1 , there are 3 predatory genes, 3 anti-prey genes and 0 neutral genes, with

(a) $C_{O 1}$ is categorised as an anti-prey behaviour,

(b) $C_{O 2}$ is considered as predatory behaviour, similar to that described in Mokhtar (2012). 


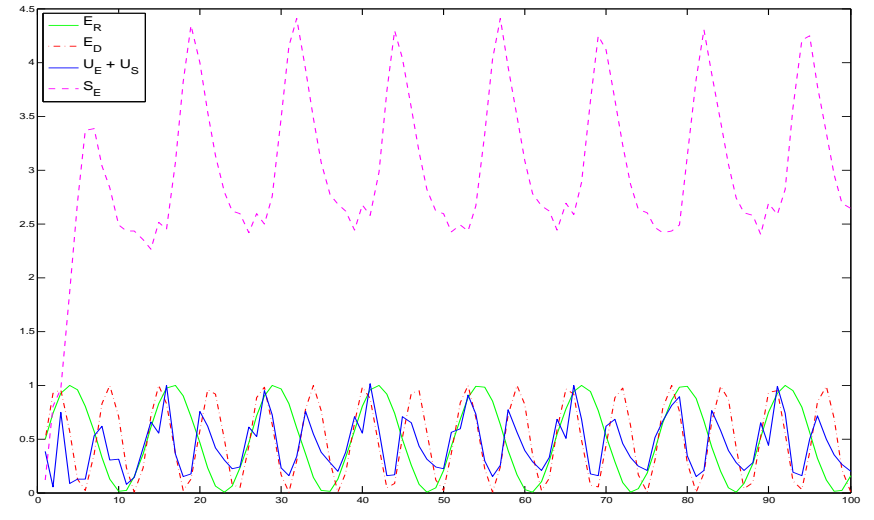

Figure 16: The $U_{E}$ and $U_{S}\left(g_{x}\right.$ and $\left.g_{x-1}\right)$ for the best evolved AGRN with $i_{t}=10$. This best evolved AGRN has the fitness values of $F_{1}=41$ and $F_{2}=0$. If based on Sec. 6.1, the AGRN consists of 4 predatory genes, 6 anti-prey genes and 2 neutral genes. This has resulted in the best evolved solution having greater tendencies to store energy at time when $E_{D}<E_{R}$, and not to use the energy to meet $E_{D}$ when $E_{D}>E_{R}$.

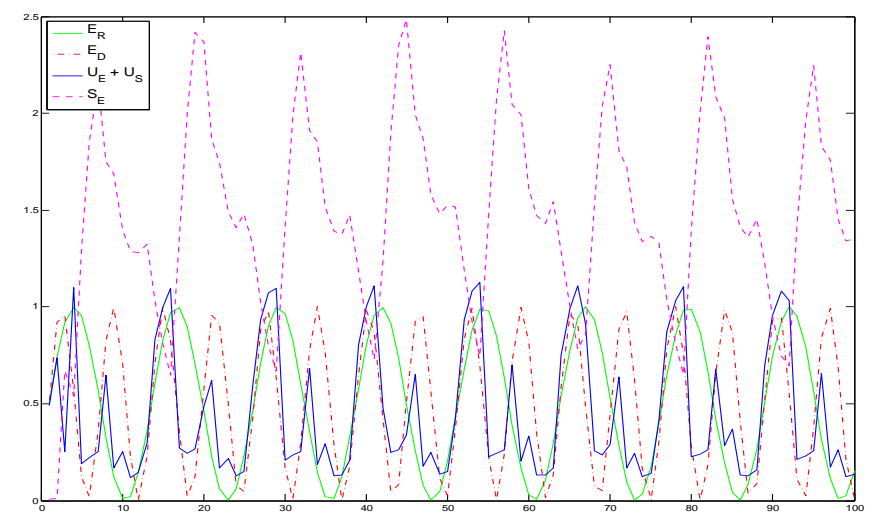

Figure 17: The $U_{E}$ and $U_{S}\left(g_{x}\right.$ and $\left.g_{x-1}\right)$ for the evolved AGRN with $i_{t}=10$, fitness values of $F_{1}=47$ and $F_{2}=0$, and an almost equal number of predatory and anti-prey genes and low number of neutral genes in its AGRN (if based on Sec. 6.1).

Figure 18 illustrates the properties of the evolved population. Figure shows that there is symmetry (equal number of predatory and anti-prey genes, with low number of neutral genes) in the best evolved AGRN. This observation is similar to other best evolved solutions. Figure 19 illustrates how well the best evolved ABN's $U_{E}+U_{S}$ meets its $E_{D}$ given the $E_{R}$.

\subsubsection{Summary of Results}

Results show that the (artificial) organisms with the best fitness values are the organisms that are in homeostasis. The organisms are in homeostasis when they have an almost equal number of predatory and anti-prey genes/chemicals in its (artificial) bio-chemical network, and with low (or zero) number of neutral genes. This observation differs from the initial heuristics made in (Mokhtar, 2012), which states that the AGRN should consist of (i) $50 \%$ predatory genes, (ii) $25 \%$ anti-prey genes,

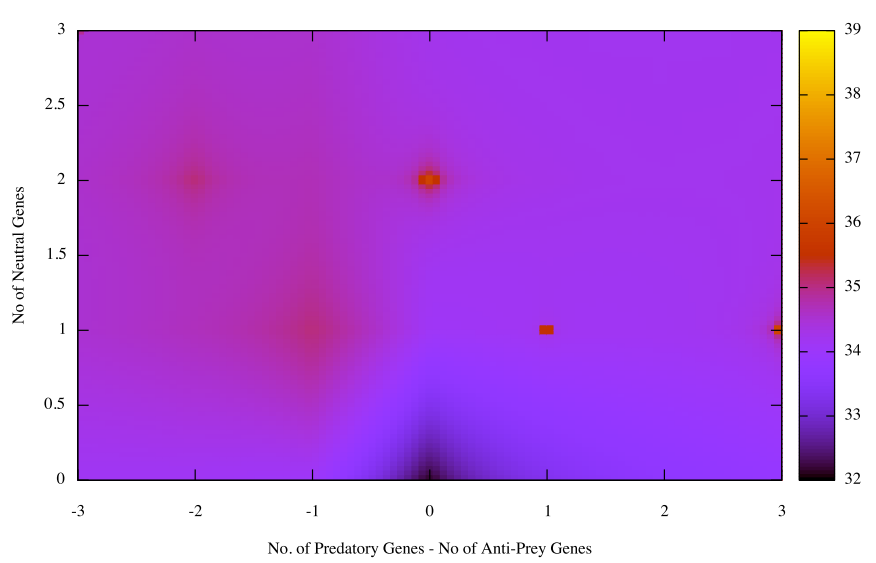

(a) Derived based on the method of analysis described in Sec. 6.1 (Algorithm 5)

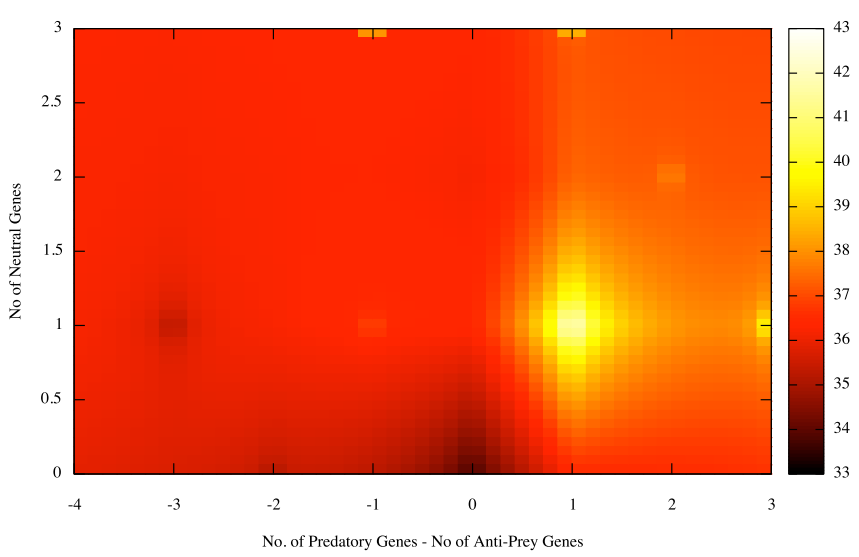

(b) Derived based on the method of analysis described in (Mokhtar, 2012) (Algorithm 4)

Figure 18: The properties of the AGRNs of the evolved ABNs. The colour values (legend) indicate the fitness value $F_{1}$ for a particular AGRN $\left(F_{2}<5\right)$. The lower the fitness value, the better the (artificial) organism. Figure shows that the best evolved organism has symmetrical property in its AGRN, with an almost equal number of predatory and anti-prey genes, and zero neutral genes (ignoring the 0.5 values in the y-axis). The best evolved ABN's $C_{O 1}$ is categorised as anti-prey behaviour, and $\mathrm{C}_{\mathrm{O} 2}$ as predatory behaviour; therefore symmetry is preserved in the organism.

and (iii) $25 \%$ neutral genes.

The new heuristics are also useful in guiding the evolution of the artificial organism. This is because better AGRNs and ABNs were found when using the fitness objectives derived from these new heuristics $\left(F_{1}-F_{10}\right)$. Further analysis of the results indicate that the fitness objectives $F_{4}$ (in Algorithm 4 ) and $F_{8}$ (in Algorithm 5) are no longer ideal for use in the evolution of the ABNs and AGRNs for the artificial organisms. This is because when two separate populations of ABN were evolved, the results of these evolution (Fig. $21-22$ ) indicate that $F_{4}$ and $F_{8}$ can delineate from finding the best solutions. 


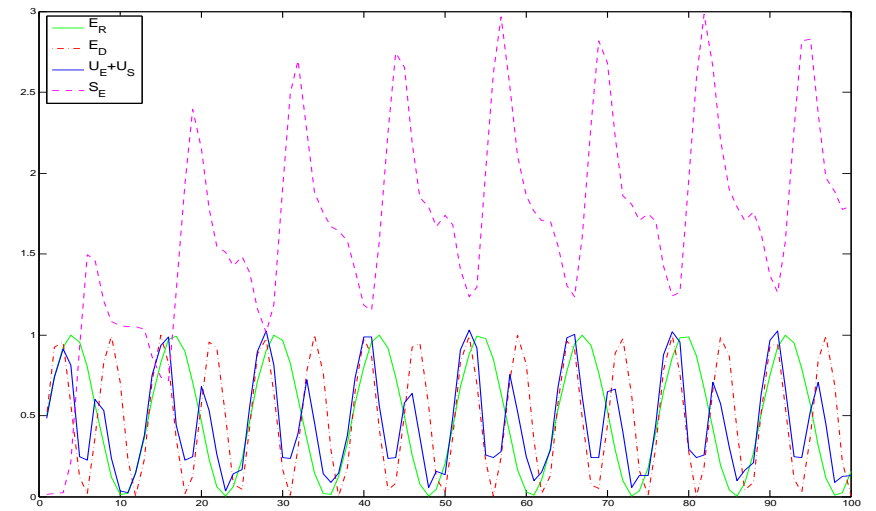

Figure 19: The $U_{E}$ and $U_{S}\left(C_{O 1}\right.$ and $\left.C_{O 2}\right)$ for the best evolved $\mathrm{ABN}$. This best evolved $\mathrm{ABN}$ has the fitness values of $F_{1}=32$ and $F_{2}=0$.

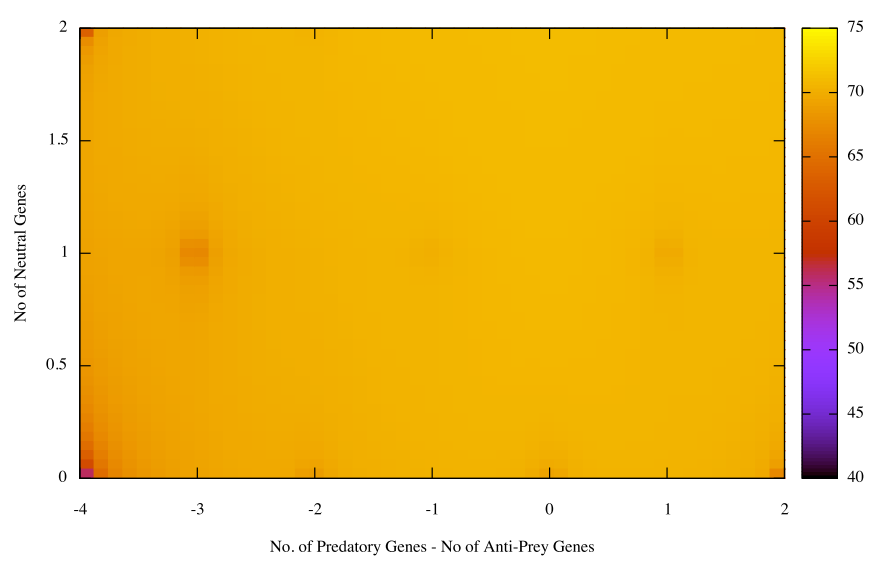

(a) Population no. 2

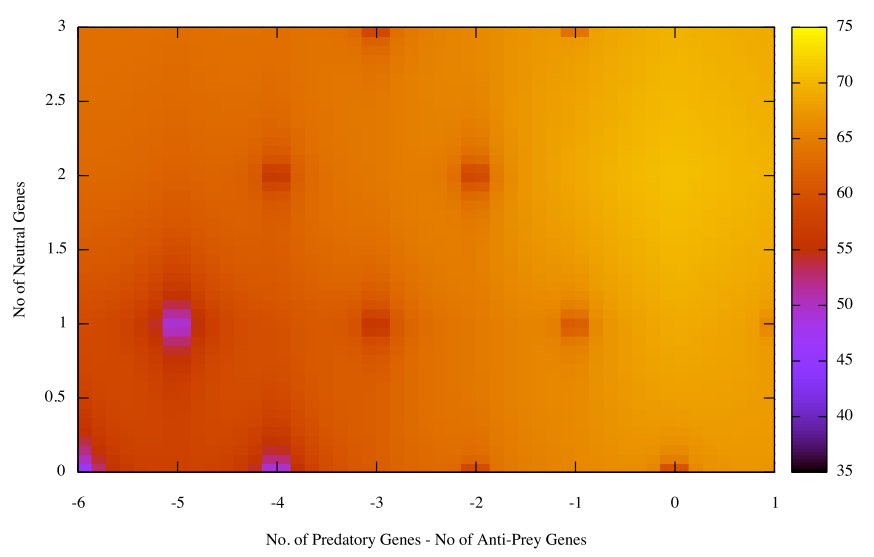

(b) Population no. 3

Figure 20: The properties of the AGRNs of a second and third separate populations of the evolved $\mathrm{ABNs}$, derived based on the method of analysis described in (Mokhtar, 2012). (Algorithm 4)

\section{Conclusions}

The results of analysis have provided useful information that can help us answer the following questions:

1. What encodes the organism's two different categories

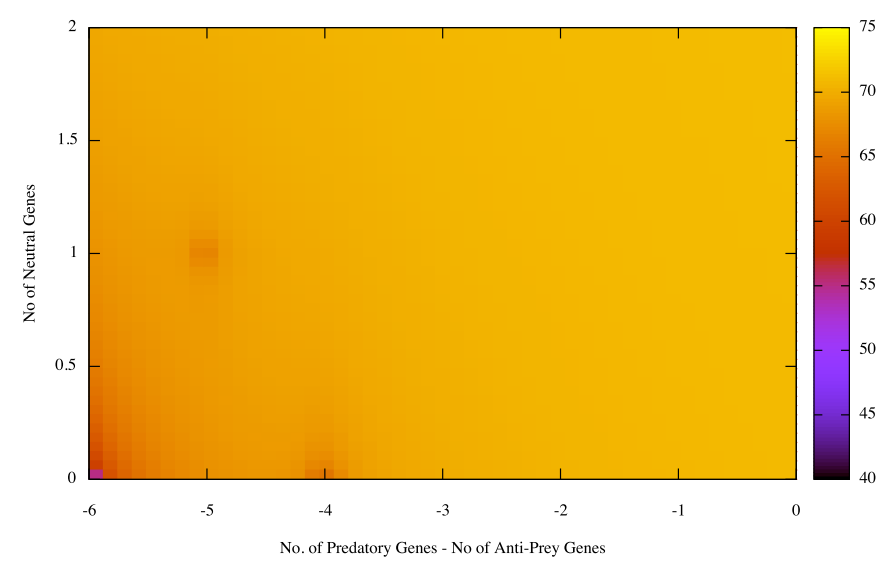

(a) Population no. 2

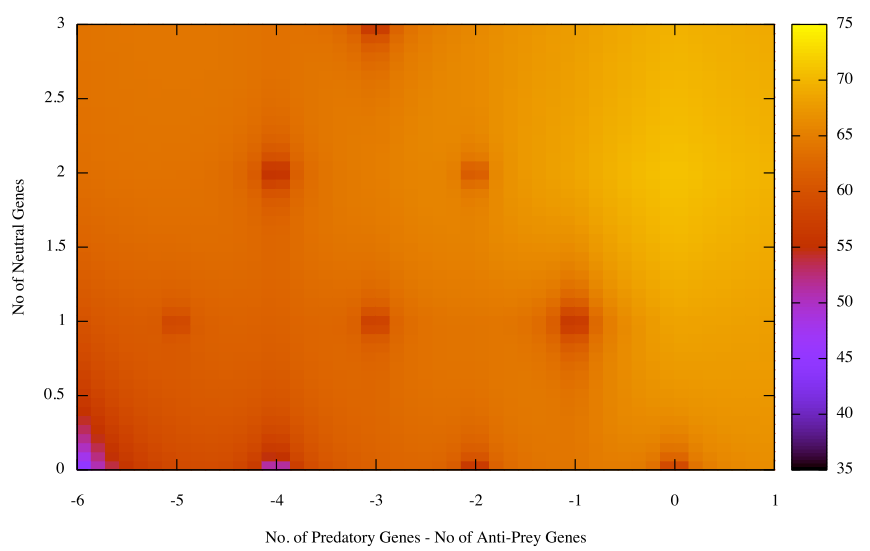

(b) Population no. 3

Figure 21: The properties of the AGRNs of a second and third separate populations of the evolved $\mathrm{ABNs}$, derived based on the method of analysis described in Sec. 6.1 (Algorithm 5). The populations were evolved for up to 1000 generations with fitness objectives $F_{1}$ to $F_{10}$. The colour values (legend) indicate the fitness value $F_{1}$ for a particular AGRN $\left(F_{2}<5\right)$. The lower the fitness value, the better the (artificial) organism. Figures show that the best evolved organism has low number of (or zero) neutral genes in its AGRN (ignoring the 0.5 values in the y-axis). Figures therefore indicate that $F_{4}$ and $F_{8}$ can delineate from finding the best solutions.

of behaviours?

(a) Can the behaviour be divided into predatory and anti-prey behaviours?

2. Is there an innate mechanism(s) that switches and resolves conflicts between the two opposing behaviours?

3. How are the organism's GRN maintains homeostasis of the organism?

4. How the organism's GRN affects the metabolic network and/or signalling network (the bio-chemical network) of the organisms in order to maintain homeostasis?

The results of analysis show that there are innate properties and mechanisms that describe and govern the switching of two opposing behaviours in an (artificial) organisms, whereby: 


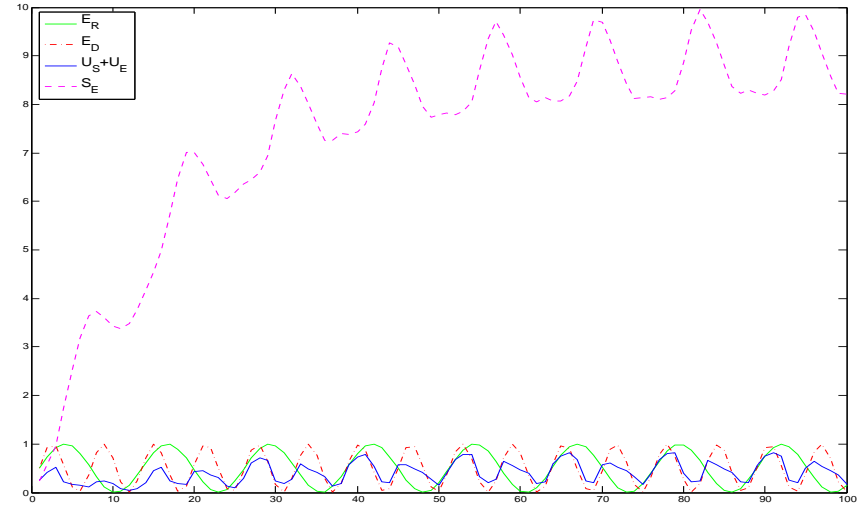

Figure 22: The $U_{E}$ and $U_{S}\left(C_{O 1}\right.$ and $\left.C_{O 2}\right)$ for the evolved ABN of population no. 3 (Fig. 20 and Fig 21), with equal number of predatory and anti-prey genes and chemicals in the AGRN and ABN, and zero neutral genes. This best evolved ABN has the fitness values of $F_{1}=50$ and $F_{2}=3$.

1. Predatory behaviours are behaviours provided by the genes or chemical outputs within its $\mathrm{ABN}$ with:

(a) $f(1) / m(1) \geq 0.6$, and

(b) $g_{x} / C_{O x}$ are constantly producing $\lambda_{x} \approx 1$.

2. Anti-prey behaviours are behaviours provided by the genes or chemical outputs with:

(a) $f(1) / m(1) \leq 0.4$, and

(b) $g_{x} / C_{O x}$ are constantly producing $\lambda_{x} \approx 0$

Results also show that the (artificial) organisms are in homeostasis when they have an almost equal number of predatory and anti-prey genes/chemicals in its (artificial) bio-chemical network, and with low number of (or zero) neutral genes/chemicals in its AGRN/ABN. This indicates that the organisms are in equilibrium.

These heuristics can and had helped in the evolution of the bio-chemical networks for the artificial organisms.

\section{Acknowledgement}

The author would like to thank Michael Lones of University of York for his help with the understanding of the coupled bio-chemical networks (artificial and biological), as well as for providing the populations of AGRNs and CABNs used for the analysis.

\section{References}

Chirikov, B. V., 1969. Research concerning the Theory of Nonlinear Resonance and Stochasticity. Tech. Report., Institute of Nuclear Physics, Novosibirsk.

de Jong H., 2002. Modeling and simulation of genetic regulatory systems: a literature review, Journal of Computational Biology 9, 67-103.

Hasty, J., Isaacs, F., Dolnik, M., McMillen, and D., Collins, J. J., 2001. Designer Gene Networks: Towards Fundamental Cellular Control, Chaos 11 (1), 207-220.

Hegner, R. E., 1985. Dominance and anti-predator behaviour in blue tits (Parus caeruleus), Animal Behaviour 33, 762-768.
Ley, R. E., Peterson, D. A., and Gordon J. I., 2006. Ecological and Evolutionary Forces Shaping Microbial Diversity in the Human Intestine, Cell 124 (4), 837-848.

Lima S. L., and Bednekoff, P. A., 1999. Back to the basics of anti predatory vigilance: can non vigilant animals detect attack?, Animal Behaviour 58, 537-543.

Lones, M. A., Tyrrell, A. M., Stepney, S., and Caves, L., 2010. Controlling Complex Dynamics with Artificial Biochemical Network, In: Esparcia-Alcaázar, A. I., et. al. (Eds.), EuroGP 2010, LNCS 6021, 159-170.

Lones, M. A., Tyrrell, A. M., Stepney, S., and Caves, L., 2011. Controlling Legged Robots with Coupled Artificial Biochemical Networks, In: Lenaerts, T., et. al. (Eds.), Advances in Artificial Life, ECAL 2011, The MIT Press, 465-472.

Lorenz, E. N., 1963. Deterministic Nonperiodic Flow, Journal of the Atmospheric Sciences, 20 (2), 1330-141,

Mokhtar, M., 2012. Understanding the Regulation of Predatory and Anti-Prey Behaviours for an Artificial Organism, In: Lones, M. A., et. al. (Eds.), IPCAT 2012, LNCS 7223, 200-211.

Popov, A., 2005. Multi-Objective Evolutionary Algorithm - Interface between Strengthen Pareto Evolutionary Algorithm 2 (SPEA2) and MATLAB, Institute of Control Systems, Hamburg University of Technology, [Online] http://pcdomino.rts.tuharburg.de/Inter/lectureE14.nsf/720dc6235ba3559b41256a1 d002a1be2/7b3ab9df2e63ca27c125739f0054fcd3?OpenDocu ment

Riechert S. E., and Hedrick A. V., 1990. Levels of predation and genetically based anti-predator behaviour in the spider, Agelenopsis aperta, Animal Behaviour 40, 679-687.

Wolf, D. M., and Eeckman, F. H., 1998. On the relationship between genomic regulatory element organisation and gene regulatory dynamics, Journal of Theoretical Biology 198, 167-186. 